\title{
Separation Criteria for Off-Axis Binary Drop Collisions
}

\author{
Mary D. Saroka ${ }^{1}$ and Nasser Ashgriz ${ }^{2}$ \\ ${ }^{1}$ United Technologies Research Center, 411 Silver Lane, MS 129-19, East Hartford, CT 06108, USA \\ ${ }^{2}$ Department of Mechanical and Industrial Engineering, University of Toronto, Toronto, ON, Canada M5S $3 G 8$
}

Correspondence should be addressed to Nasser Ashgriz; ashgriz@mie.utoronto.ca

Received 15 December 2014; Accepted 14 April 2015

Academic Editor: Robert Spall

Copyright (C) 2015 M. D. Saroka and N. Ashgriz. This is an open access article distributed under the Creative Commons Attribution License, which permits unrestricted use, distribution, and reproduction in any medium, provided the original work is properly cited.

Off-axis collisions of two equal size droplets are investigated numerically. Various governing processes in such collisions are discussed. Several commonly used theoretical models that predict the onset of separation after collision are evaluated based on the processes observed numerically. A separation criterion based on droplet deformation is found. The numerical results are used to assess the validity of some commonly used phenomenological models for drop separation after collision. Also, a critical Weber number for the droplet separation after grazing collision is reported. The effect of Reynolds number is investigated and regions of permanent coalescence and separation are plotted in a Weber-Reynolds number plane for high impact parameter collisions.

\section{Introduction}

Many engineering applications and natural process involve drop collisions in which the final outcome has a direct impact on the eventual success or failure of the application or process. In sprays, for example, the behavior is characterized by many small liquid drops with a particular size distribution and with each individual drop moving along a particular trajectory with a prescribed velocity. By singling out and focusing on just two drops within the spray, one can readily see that the interaction between these drops plays a part in the overall evolutional characteristics of the spray. One can further imagine that the likelihood of two drops colliding along the same axis of trajectory is less likely to occur than the situation in which the two drops collide along different trajectories. It is the latter case, typically referred to as off-axis collisions, that is the subject of this investigation.

Over the years, many researchers have studied the collision dynamics of the binary drops. The early studies on drop collision were motivated by understanding of the physical processes that occur during rain fall [1-12]. Rain drops may collide with each other and break into smaller drops or coalesce and generate larger drops. For instance, Park [5] did experiments on water drops in humid environment to determine conditions for the drop separation after collision. Several phenomenological models for the drop separation after collision are provided, for instance, the rotational energy model [7] and the variation principal model for the minimum potential energy for the stretching separation [12]. The later studies were motivated by drop collision in various spray systems [13-28]. Collisions of many other liquids, such as hydrocarbons [13-18], heavy oils [19, 20], and mercury [21], in addition to water, have also been studied. The bulk of quantitative information on the drop collision is obtained through experiments [21-28], while numerical studies are used to provide detailed understanding of this complex process. The front tracking techniques [29-32] and a combination of level set and VOF methods are used to solve the dynamics of the free surfaces [33-40]. A detailed review of the droplet collision process is provided by Brenn [41]. Review of this body of research indicates that, depending upon the initial energy of the two drops and the angle at which they collide, different types of outcomes will occur. These outcomes can be categorized as bouncing, partial coalescence, permanent coalescence, separation, or shattering. There are also several subcategories for each outcome. Of these five, permanent coalescence and separation are the most frequently observed outcomes, while the other three, namely, bouncing, partial coalescence, and shattering, represent special cases in which the drops have either very small velocities, large size differences, or very high velocities.

The present study is on the off-axis collision of two equal size droplets. This study is a continuation of a previous 


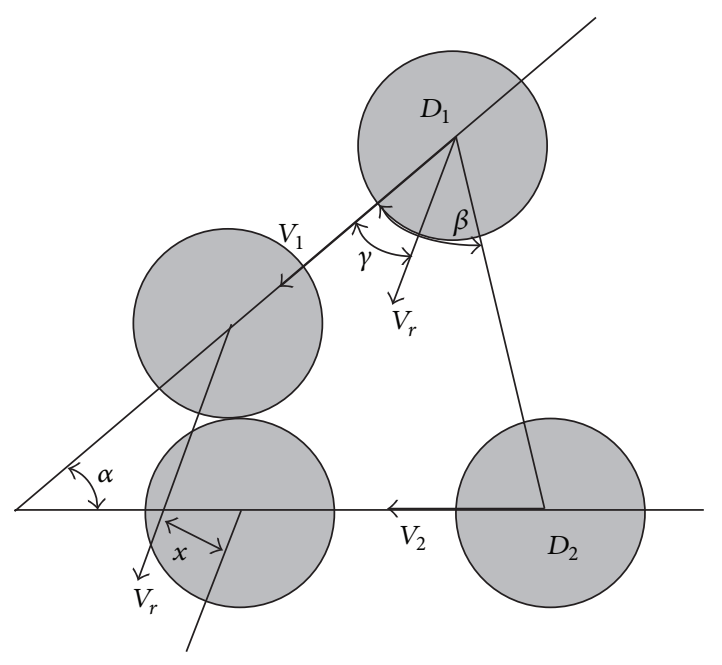

FIGURE 1: General orientation and parameter definition for binary droplet collisions.

study by the authors on the head-on collision of two drops [40]. Droplet collisions in dynamically inert surroundings are considered. Therefore, bouncing collisions, which are due to air entrapment between the two approaching droplets, are not observed. In the next sections, first the problem statement and the numerical methods are described, followed by the results and analysis.

\section{Problem Statement}

A general schematic depicting the positions and velocity vectors of two drops just prior to contact is shown in Figure 1. Suppose that one of the drops has a velocity defined as $V_{1}$, while the other has a velocity of $V_{2}$. These individual velocities are used to define a relative velocity, $V_{r}$, of the interacting drop. This is done by combining the drop velocities and their impact angle, $\alpha$, through the following relation: $V_{r}=$ $\sqrt{V_{1}^{2}+V_{2}^{2}-2 V_{1} V_{2} \cos \alpha}$. Another important parameter necessary to define a particular collision involves the distance between the drops' centers. This distance is referred to as the impact parameter, noted as $x$. As the impact parameter increases the amount of "interaction" is reduced, while the converse is true. Using this information, it is convenient to define various dimensionless numbers to represent the phenomena under consideration. Three dimensionless parameters, namely, a Weber number, a nondimensional impact parameter, and a drop diameter ratio, are defined based on the drop diameter, liquid density, $\rho$, and the coefficient of surface tension, $\sigma$ :

$$
\begin{aligned}
\mathrm{We} & =\frac{\rho D V_{r}^{2}}{\sigma}, \\
X & =\frac{x \sin |\beta-\gamma|}{D},
\end{aligned}
$$

where

$$
\sin \gamma=\frac{V_{1}}{V_{r}} \sin \alpha .
$$

In addition, Reynolds and Ohnesorge numbers are used to correlate effect of viscosity, $\mu$, and its relationship with surface tension:

$$
\begin{aligned}
& \mathrm{Re}=\frac{\rho D V_{r}}{\mu}, \\
& \mathrm{Oh}=\frac{\sqrt{\mathrm{We}}}{\mathrm{Re}}=\frac{\mu}{\sqrt{\rho D \sigma}} .
\end{aligned}
$$

The Ohnesorge number is unique in the fact that it is only a function of fluid properties and the drop size, while both the Weber and Reynolds numbers are functions of the fluid properties, drop geometry, and impact velocity. In the present study, We, Re, and Oh numbers are calculated based on the liquid properties.

In order to discuss different types of collision outcomes, some collision images obtained by Ashgriz and Poo [22], but not published earlier, are provided here. The experimental setup and conditions are all as described in Ashgriz and Poo [22] and will not be repeated here. Figure 2(a) shows a close to head-on collision of two water droplets. After the collision, drops spread radially on each other until the kinetic energy of the drops is transformed into the surface energy. The surface energy is large enough to push back the fluid and form a ligament. The ligament stretches until it breaks into two droplets. This is referred to as a "reflexive separation." Ashgriz and Poo [22] have provided a theoretical model to predict the onset of this type of separation. If the collision impact parameter is increased enough, the impact process becomes complicated. Part of the drop fluid goes through the spreading and reflexive action, and another part of the drop fluid tends to stretch and pull the drops away from each other. If the collision kinetic energy is just enough to have reflexive separation, as shown in Figure 2(a), then a small increase in the impact parameter reduces this reflexive energy, and drops may not separate and stay coalesced. This process is shown in Figure 2(b), where the coalesced drop goes through significant deformation and oscillations, but drops remain coalesced. If the impact parameter is further increased, then the part of each drop which remains uninteracted with the other drop tends to stretch and pull the drops apart. This is observed in Figures 2(c), 2(d), and 2(e), and it is referred to as stretching separation. The present study only addresses stretching separation.

\section{Numerical Methodology}

We consider two drops composed of the same fluid with constant properties. The flow inside the drops is solved using the standard conservation of mass and momentum equations:

$$
\begin{aligned}
\nabla \cdot \mathbf{V} & =0 \\
\rho \frac{\partial \mathbf{V}}{\partial t}+\rho(\mathbf{V} \cdot \nabla) \mathbf{V} & =-\nabla p+\mu \nabla^{2} \mathbf{V}+\mathbf{F}_{\mathbf{b}}
\end{aligned}
$$




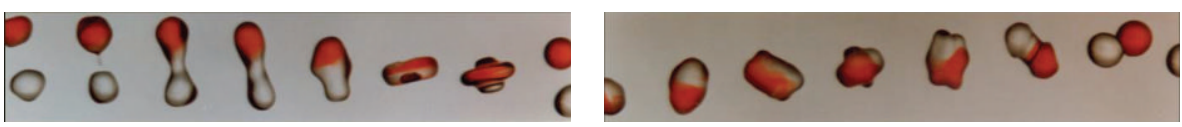

(a)

(b)

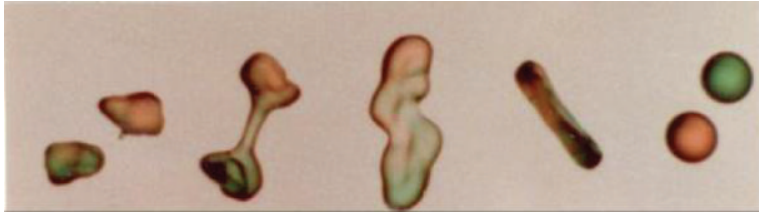

(c)

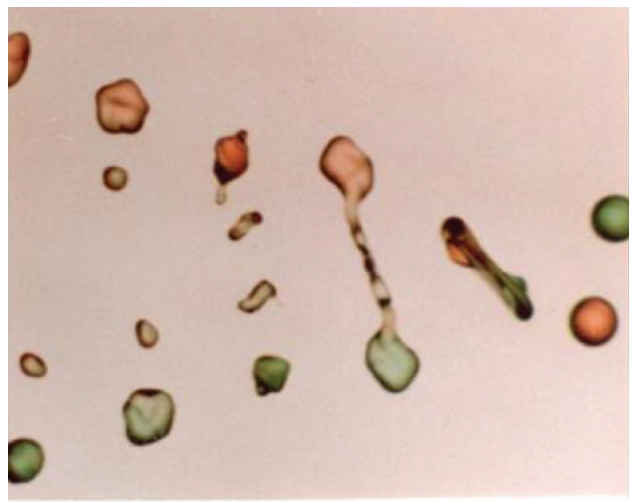

(e)

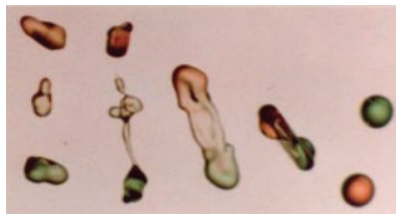

(d)

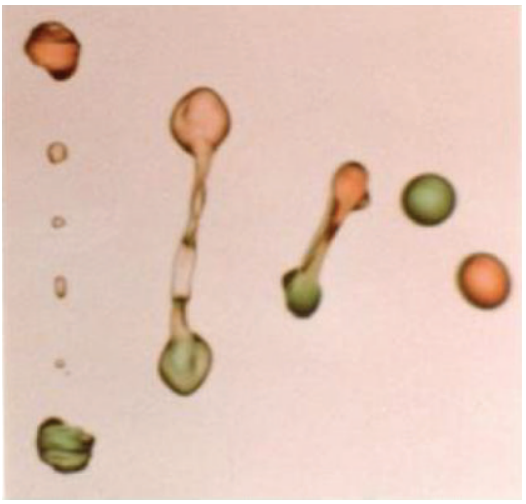

(f)

FIGURE 2: Binary collision of water droplets. (a) Reflexive separation due to near head-on collision; (b) coalescence collision; (c) separation collision resulting in two drops; (d) separation collision resulting in three drops; (e) separation collision resulting in four drops; and (f) separation collision resulting in six drops.

where $\mathbf{V}$ is the velocity vector, $p$ is the pressure, and $\mathbf{F}_{\mathbf{b}}$ is any volume force acting on the drops. At the free surface, the boundary condition requires that both mass and momentum are conserved. For drops surrounded by a less dense/viscous fluid or gas, the viscous stresses at the surface are zero. Additionally, assume a constant surface tension coefficient allows the stress boundary condition to reduce to Laplace's equation $p_{s}=\sigma \kappa$, where $p_{s}$ is the pressure jump across the surface and $\kappa$ is the surface curvature. Details of the numerical simulation have been discussed in earlier publications $[40,42,43]$ and are not repeated here.

Two drops with a prescribed distance separating their respective centers are considered. In all cases, this distance was defined to occur in one direction only. That is to say that for drops translating in one direction (i.e., $z$-direction) their centers were placed in the same plane (called the defining plane) composed of this direction and one of the other two remaining directions (i.e., $y-z$ or $x-z$ plane). The third direction (i.e., $x$ or $y$, resp.) was held constant for both drops. One might imagine that this third direction could also represent an off-set, thus indicating a second impact parameter. However, this case was not considered during this investigation. Figure 3 provides an example of the defining plane for an off-axis collision. This plane represents the $y$ $z$ plane and is useful in observing the internal flow patterns as the two drops collide and evolve. Although similar planes such as $X-Z$ or $X-Y$ planes could be defined, they would only represent a specific region of the collision and are therefore not as comprehensive.

The distance between the drops' centers was used to compute the impact parameter. For this investigation, impact parameters ranging from 0 to 1 were considered, with the bulk of the simulations focusing on collisions for $X>0.5$ (i.e., high impact parameters). In order to cause the drops to collide, each drop was given a nonzero initial velocity. These individual drop velocities were subsequently used to compute the relative velocity, $V_{r}$. All simulations were conducted using water drops for Weber numbers ranging from 10 to 60 and Reynolds numbers ranging from 135 to 1639 . Since various drop sizes ranging from $10 \mu \mathrm{m}$ to $2 \mathrm{~mm}$ were used in the course of this investigation, a wide range of relative velocities ranging from $0.5 \mathrm{~m} / \mathrm{s}$ to $21 \mathrm{~m} / \mathrm{s}$ were considered. For very small drops of $10 \mu \mathrm{m}$, relative velocities as high as $21 \mathrm{~m} / \mathrm{s}$ were used, while for the larger drops of $2 \mathrm{~mm}$, velocities less than $1 \mathrm{~m} / \mathrm{s}$ were predominately used. The fluid properties, density, $\rho$, viscosity, $\mu$, and the coefficient of the surface tension, $\sigma$, were kept constant at $1000 \mathrm{~kg} / \mathrm{m}^{3}, 10^{-6} \mathrm{~m}^{2} / \mathrm{s}$, and $0.073 \mathrm{~N} / \mathrm{m}$, respectively.

In order to resolve the flow within the drops, 10 cells per radius were used. A computational grid of $70 \times 70 \times 180$ cells in the $x$-, $y$-, and $z$-directions was used to allow the collision to evolve without hitting any walls. The use of 180 cells in the $z$-direction was necessary, since this was the primary direction in which the drops approached each other. Higher 


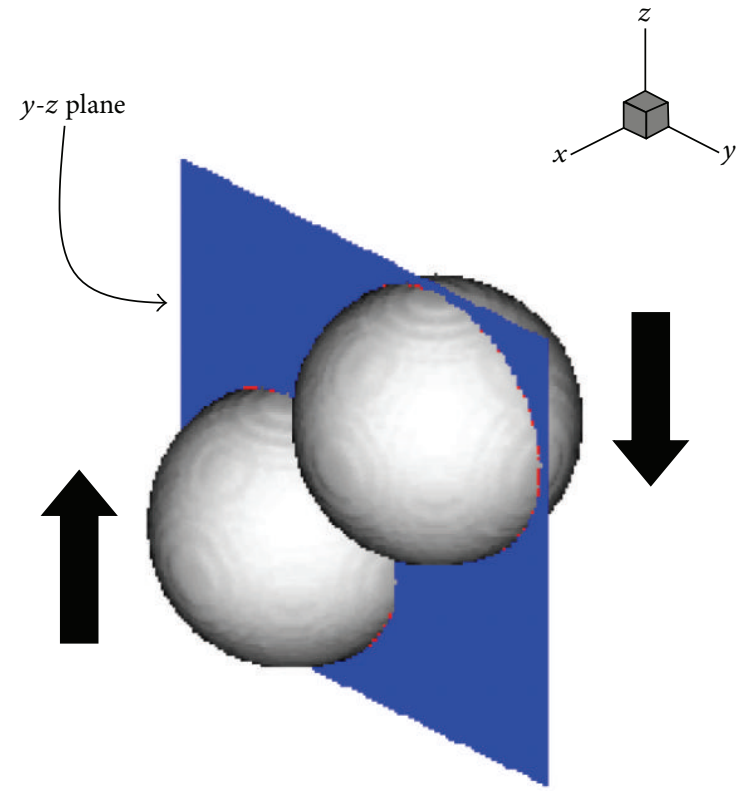

FIGURE 3: Definition of the $y-z$ plane. Original two drops translating in the $\pm z$-direction.

Weber numbers than those considered were not simulated, since these types of collisions required a larger computational grid to fully capture the evolutional dynamics of the collision.

\section{Results and Discussion}

Figures 4-6 show typical surface evolutions of water drops colliding at impact parameters greater than zero. Figure 4 shows a coalescence collision of two $300 \mu \mathrm{m}$ drops colliding at $\mathrm{We}=25, \mathrm{Re}=740$, and $X=0.7$. Figure 5 shows results for a similar case as in Figure 4 but with a slightly larger relative velocity, where two $300 \mu \mathrm{m}$ drops collide at $\mathrm{We}=30, \mathrm{Re}=$ 811 , and $X=0.7$. It is shown that the boundary between the coalescence and separation at the impact parameter of $X=$ 0.7 occurs at a Weber number between $25<\mathrm{We}<30$. A small increase in the impact We number causes the drops to separate after collision. Comparing Figures 4 and 5 with each other, it is clear that, in Figure 4, the surface energy pulls the ligament formed at stage 5 back together, and drops coalesce. In Figure 5, the ligament formed at stage 10 is longer than that in Figure 4. In this case, the pinching effect, which is governed by the Rayleigh type effect, is faster than the forces that tend to coalesce the drops. Therefore, the ligament in Figure 5 breaks before it comes back forming a coalesced droplet.

A general description of an off-axis collision process is as follows. As the drops come into contact with one another, a small region of interaction develops, while the remaining portion of the drops continues on their original course. This stretches the collided region. As the combined mass continues to stretch, the interaction region allows a portion of the fluid at the ends to drain back towards the middle of the system. This in turn causes the entire mass to rotate in a clockwise direction when the top drop is to the right of the

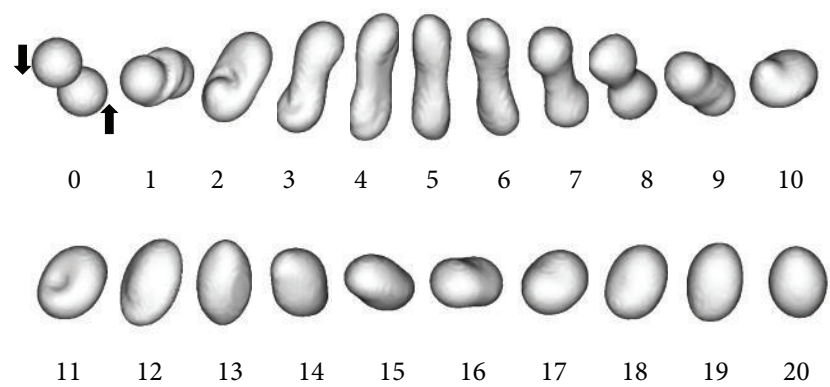

FIGURE 4: Coalescence collision of two $300 \mu \mathrm{m}$ water droplets with $\mathrm{We}=25, \mathrm{Re}=740$, and $X=0.7$. Numbers in the figure indicate time $=$ $(0.1 \mathrm{~ms}) i$, where $i=$ image number.

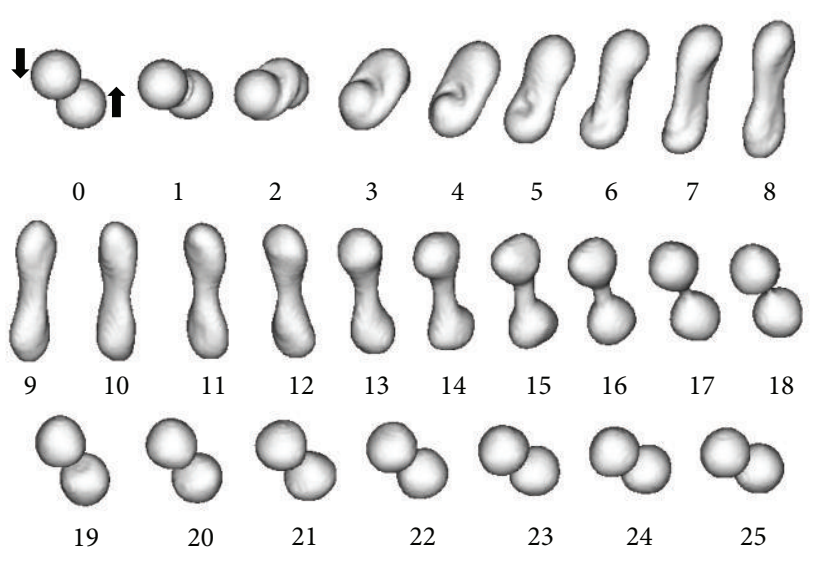

FIGURE 5: Separation collision of two $300 \mu \mathrm{m}$ water droplets with $\mathrm{We}=30, \mathrm{Re}=810$, and $X=0.7$. The numbers in figure indicate time $=$ $(0.05 \mathrm{~ms}) i$, where $i=$ image number.

bottom drop (opposite orientation results in counterclockwise rotation). Fluid within the mass continues to adjust itself in this manner until a liquid bridge between the two ends is formed. Depending upon the amount of initial energy present in the system and the magnitude of the impact parameter, the rotating mass will either contract resulting in permanent coalescence or separate without or with satellite drops. Figure 6 shows collision of two $700 \mu \mathrm{m}$ drops colliding at $\mathrm{We}=60, \mathrm{Re}=1751$, and $X=0.7$. In this case, a long ligament is formed, which later results in a satellite droplet in addition to the two main droplets. It can be seen in images 16 to 24 that the rate of contraction of the inner ligament is faster than the end pinching of the drops. Therefore, only one satellite is formed. If the inner ligament was slightly longer, end pinching could result in three satellite droplets.

4.1. Internal Flow Patterns. There has been a lot of debate about the existence of internal circulations in droplets of a spray. Since it is very difficult to see the internal flow of a moving droplet, the main way to determine this effect has been through numerical modeling. The present study puts forward a concept for the formation of the internal circulations in the droplets. Figure 7 shows the internal flow patterns for the drop collision of Figure $6(\mathrm{We}=60$, 

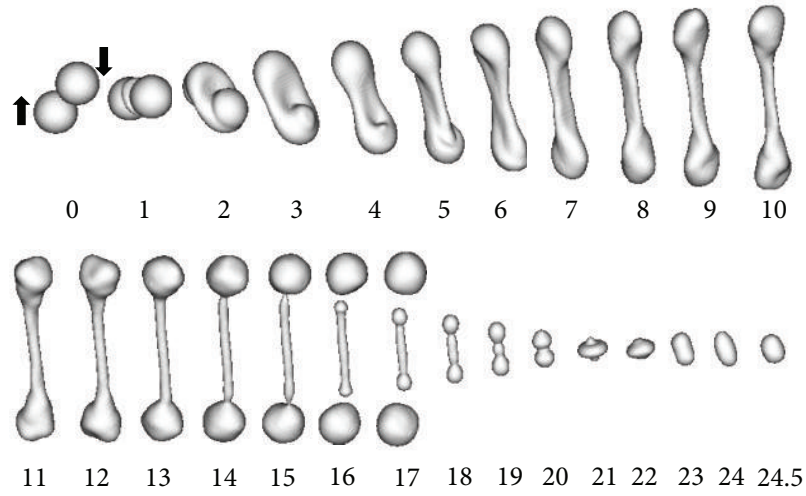

FIGURE 6: Stretching separation with one satellite droplet for the collision of two $700 \mu \mathrm{m}$ water droplets with $\mathrm{We}=60, \mathrm{Re}=1751$, and $X=0.7$. The numbers in the figure indicate time $=(0.2 \mathrm{~ms}) i$, where $i=$ image number.

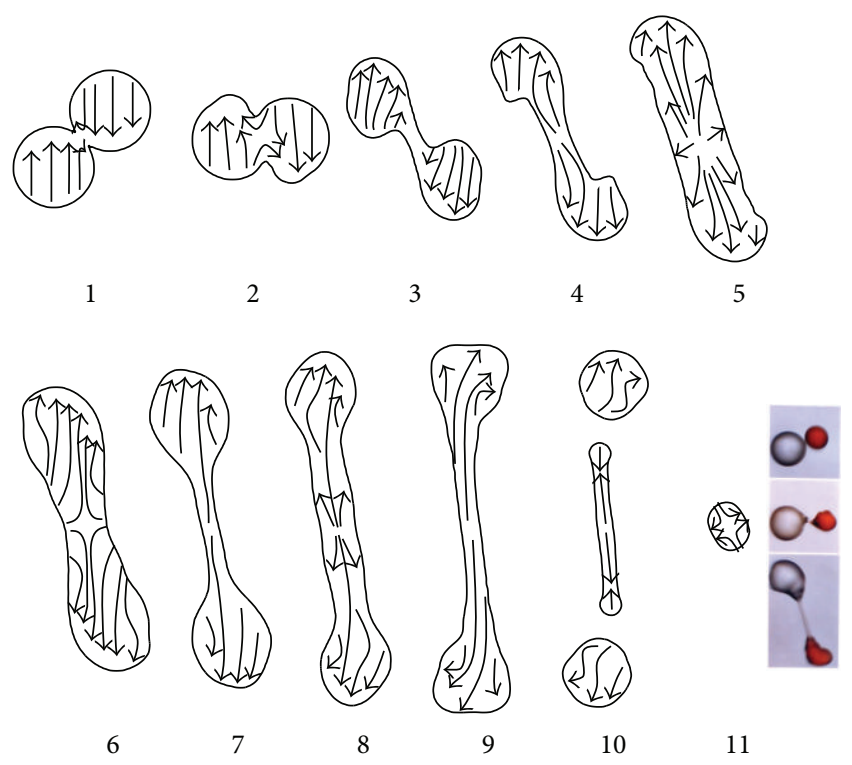

FIGURE 7: Internal flow patterns for the $y-z$ plane. Results are for a binary water drop collision with a diameter of $700 \mu \mathrm{m}$ and $\mathrm{We}=60$, $\operatorname{Re}=1751$.

$\operatorname{Re}=1751$, and $X=0.7)$ and for the plane described in Figure 3. The first image in Figure 7 shows the two drops just after contact. Immediately after the collision a small region of interaction is produced. In this region the fluid is redirected away from the collision point. As this occurs, the outer (or noninteracting) portion continues to translate unimpeded in the original direction (as shown in images 2-4). In the fifth image, in the center of the combined mass some of the flow begins to be redirected "radially" while the bulk of the motion still occurs in original translating direction. However, surface tension compensates and the radial flow is redirected, thus causing the overall flow to be directed upward/downward and away from the center. This action causes the stretching phenomena observed in off-axis collisions. The bulk of the fluid eventually ends up in the bulbous ends as indicated by
TABLE 1: Upper critical Weber numbers for two equal size water droplets $(X=1)$.

\begin{tabular}{lcc}
\hline $\begin{array}{l}\text { Diameter } \\
\mu \mathrm{m}\end{array}$ & $\mathrm{We}_{\mathrm{cr}}$ & $\mathrm{Re}$ \\
\hline 10 & 32.5 & 154 \\
75 & 22 & 347 \\
150 & 21 & 480 \\
300 & 20.3 & 665 \\
700 & 18.3 & 966 \\
2000 & 18 & 1622 \\
\hline
\end{tabular}

the streamlines in images 7-9. Image 10 indicates that separation has occurred with the production of two parent drops and a ligament. It is interesting to note several observations at this point. First, review of early images does not provide any evidence of the pinching mechanism in this defining plane. Therefore, the pinching phenomena are the result of radial type flow. Second, the parent drops exhibit flow patterns representing translating flow. Unlike the scenario for the head-on collisions [40] these parent drops reflect similar flow patterns of the original two drops which causes them to continue to translate after separation occurs. Lastly, the ligament collapses into a satellite drop producing the symmetrical flow pattern observed. Therefore, main droplets after collision have unidirectional internal flows, whereas satellite droplets may have multidirectional and internal flow circulations.

4.2. The Upper Boundary between Coalescence and Separation. The upper boundary between a coalescence and separation collision occurs for grazing collisions, that is, $X=1$. As soon as the surfaces of two drops touch, the liquid flows between them. Depending on the collision conditions, the two drops may touch and temporarily coalesce, and then separate, or remain coalesced. The We number corresponding to this condition is referred to as the upper critical We number, $\mathrm{We}_{\mathrm{cr}}$. Table 1 shows the numerically determined upper critical Weber number for equally sized water drops as a function of drop diameter. It is clear that the upper critical Weber number increases with decreasing diameter. The pressure inside smaller drops is larger than those in larger drops; therefore, the flow is pushed into the liquid bridge between the two drops faster in the smaller drops than for the larger drops. The larger the mass of the bridge, the more difficult it is to break the drops. Therefore, the separation occurs at higher Weber numbers for the smaller drop collisions. The curve fit to the data of Table 1 is $\mathrm{We}_{\mathrm{cr}}=$ $-2.7 \ln D+36$. As the drop size increases, the effect of change in drop size becomes less important. The upper critical We number approaches $\mathrm{We}=18$ for large drop sizes.

4.3. Stretching Separation Boundary. The boundary between permanent coalescence and separation for off-axis collision of two equal size droplets for different collision impact parameters is obtained. Figure 8 presents the results of this investigation for equal size drop collisions with a diameter 


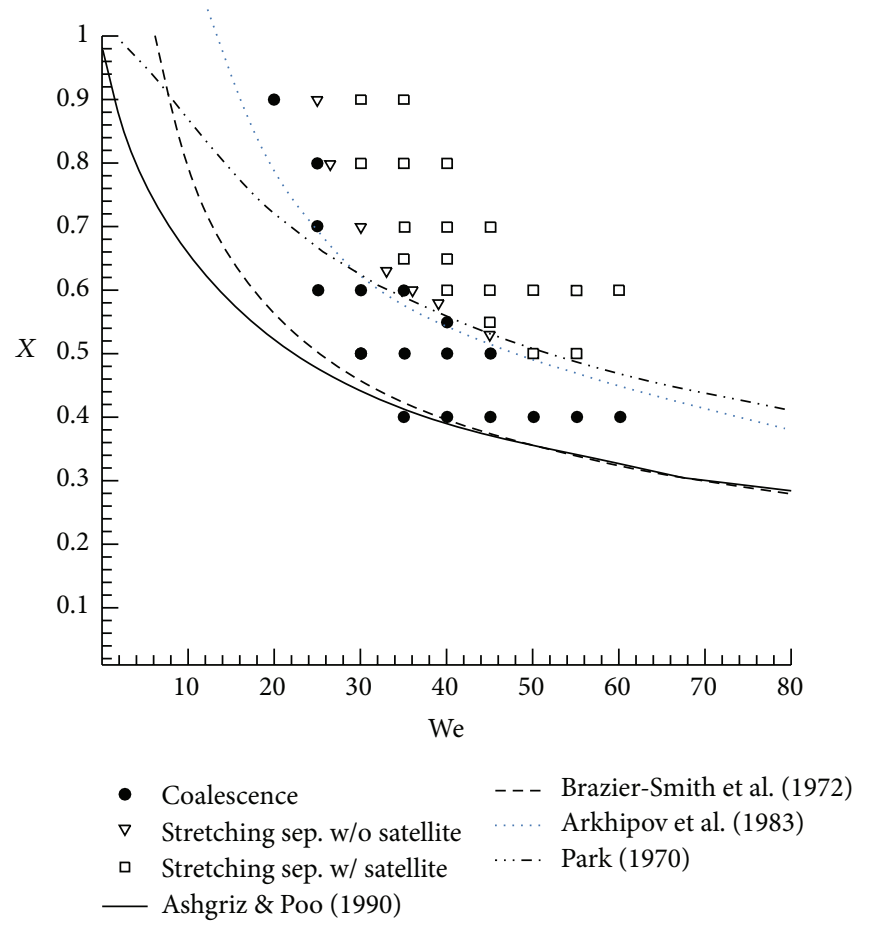

(a)

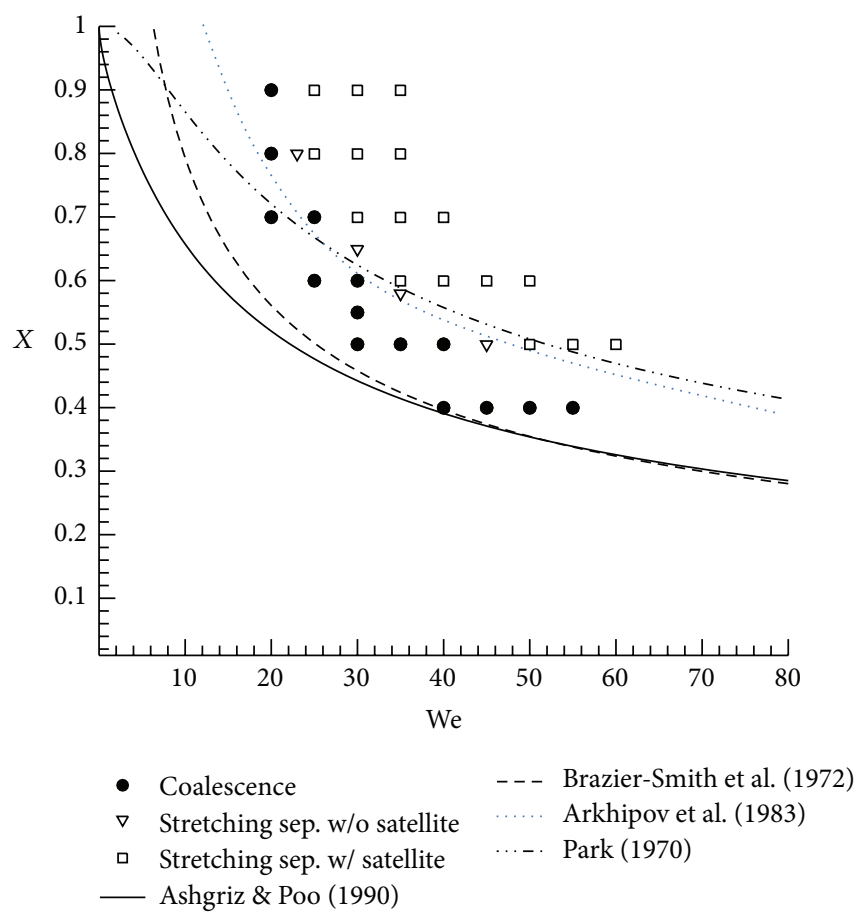

(b)

Figure 8: Coalescence-fragmentation curves: (a) $300 \mu \mathrm{m}$ drop collisions and (b) $700 \mu \mathrm{m}$ drop collisions. Symbols represent numerical data while lines represent boundary models presented by previous researchers.

of $300 \mu \mathrm{m}$ (Figure $8(\mathrm{a})$ ) and $700 \mu \mathrm{m}$ (Figure 8(b)). Outcomes of permanent coalescence, separation without satellite production, and separation with one satellite produced are plotted for a range of Weber numbers and impact parameters. Also included in the figures are the boundaries defined by the analytical models proposed by Ashgriz and Poo [22], Arkhipov et al. [12], Brazier-Smith et al. [7], and Park [5]. The model by Ashgriz and Poo [22] is referred to as AP/EXP model for the remainder of the discussion. Similarly, the other models are referred to as A, B-S, and P, respectively. The results show that, for a constant impact parameter, as the Weber number is increased, the outcome changes from coalescence to separation without satellites to separation with a satellite. Similarly, as the Weber number is held constant, the same progression is observed provided there is enough energy initially present in the drops (i.e., at low Weber numbers, regardless of impact parameter, coalescence will always occur).

The numerical division between the coalescence and separation occurs at higher Weber and impact parameter combinations than those observed by the AP/EXP and B-S models. A possible explanation for this behavior is attributed to the existence of trapped air in the gap between the colliding drops. In the experiments, the impact parameter is determined by measuring the lines of trajectory and the corresponding separating angle [22]. Depending upon when this is done (i.e., a few diameters from the drop generators or just prior to impact) the value of the impact parameter can be quite different. As the two drops travel through air, their trajectories remain relatively constant. However, as they come closer and closer, the surrounding gas becomes trapped in between them, which results in the slippage of the drops on each other. This may alter the original impact parameter based on the drop trajectories prior to collision. The effect of such a slippage is shown in Figure 9 by a shift in data. In this figure, the solid line represents the boundary using the AP/EXP model while the symbols represent the numerical results for the stretching separation without satellite production. The dashed line represents a linear translation of the $\mathrm{AP} / \mathrm{EXP}$ model and is meant to convey the concept of impact parameter slippage. For example, at $\mathrm{We}=31$, the impact parameter on the AP/EXP boundary is $X=0.44$, while that numerically is $X_{s}=0.64$. Thus, due to the presence of the trapped air, the impact parameter at the time of collision could actually be higher than that previously thought.

The presence of the trapped air has three different effects on the drops, which ultimately cause an increase (or slippage) in the effective impact parameter. These effects are (i) altered trajectories, (ii) altered internal flow fields, and (iii) shape deformation. The trapped air conveys an external force on the drops, which may alter their trajectories, as shown in Figure 10. The second effect is shown in Figure 11, where drops moving in air may develop internal flows. The internal flow changes the drop dynamics after collision and may change the collision outcome. Finally, the surrounding air may cause drop deformation, as shown in Figure 12. As a result of this deformation the region of contact may increase as opposed to the case in which the drops stay 


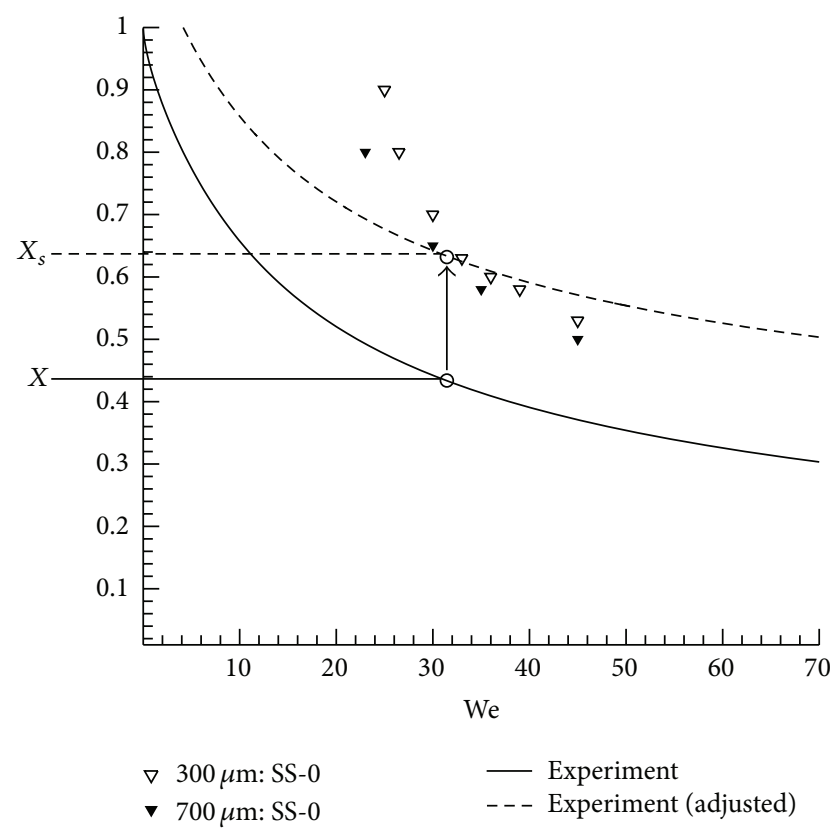

FIGURE 9: Impact parameter deviation for the same Weber numbers. $X$ is a point on the experimental boundary, while $X_{s}$ represents the impact parameter observed numerically. The solid line represents the boundary model by Ashgriz and Poo [22], while the dashed line represents a linear translation added to their model.

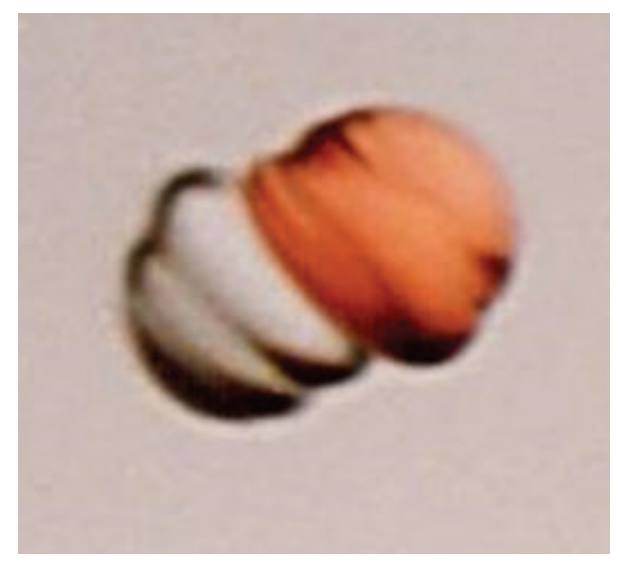

FIGURE 10: Effect of trapped air between colliding drops. Experimental photo from Ashgriz (unpublished material from Ashgriz and Poo [22]). The trapped air alters the collision impact parameter based on droplet trajectories.

perfectly spherical. This increased contact region may result in a larger interaction region and thus more mixing. It is, therefore, concluded that, in determining the collision impact parameter, one has to be worried about the slippage effect that may occur due to the air entrapment.

Figure 8 also compares the numerical results with models by Arkhipov et al. (A) [12] and Park (P) [5]. Arkhipov assumed that after the collision a spherical drop is formed that rotates with a constant angular velocity, while Park assumed that the combined mass rotates as two connected spheres. The numerical results, as evident in Figure 5, show that, for the drops to separate after collision, they rotate as two drops that are connected, and not as a combined spherical mass. Therefore, Park's model is closer to numerical observation of the collision dynamics. However, the drop shapes and masses are altered and are not exactly the same as the original droplets. This becomes particularly true as the Weber number is increased. However, at low Weber numbers, We $<25$, the numerical results do not correlate with any of the models considered. This is most likely explained by an incorrect assessment of the amount of angular velocity produced during the evolution. One could imagine that the amount of angular velocity would in fact not be constant (as assumed by Arkhipov et al.) and be a function of the impact parameter instead.

4.4. Deformation. The deformation variations in each direction for an off-axis collision of two water drops with a Weber number of 30 and a Reynolds number of 810 are provided in Figures 13-16. For clarity, the deformations are grouped according to either low impact parameters, $X<0.5$, or high impact parameters, $X>0.5$. It is convenient to nondimensionalize the amount of deformation by dividing by the drop's initial diameter. In doing so, the following definitions are used to represent the dimensionless deformation in each direction:

$$
\begin{aligned}
& \eta=\frac{L_{x}}{D}, \\
& \gamma=\frac{L_{y}}{D}, \\
& \psi=\frac{L_{z}}{D},
\end{aligned}
$$

where, $L_{x}$ is the amount of deformation in the $x$-direction, $L_{y}$ is the amount of deformation in the $y$-direction, and $L_{z}$ is the amount of deformation in the $z$-direction. Since offaxis collisions occur on different trajectories, the evolution process occurs in all three dimensions, resulting in separate behavior for each direction. In the next three subsections, the behavior in each direction is analyzed.

As observed with head-on collisions [40], three major periods of the collision process are spreading and production of a disk, period I, contraction of the disk, period II, and stretching along the collision axis ( $z$-direction), period III.

4.5. $x$-Direction (Minor Axis 1). Figure 13 contains the dimensionless deformation in the $x$-direction. Review of this figure during period I provides the following information. First, it is observed that, at $t^{*}=0, \eta$ increases as the impact parameter, $X$, increases. This behavior is a direct consequence of the impact parameter definition, since the drop's separating distance is defined in this direction. Second, as the impact parameter is increased the amount of spread decreases. Therefore, as the region of interaction becomes smaller due to the increased impact parameter, less fluid is allowed to flow in this direction. Additionally, at $X \sim 0.6$, no spread is observed and the deformation actually becomes drainage or contraction for higher impact parameters. This 


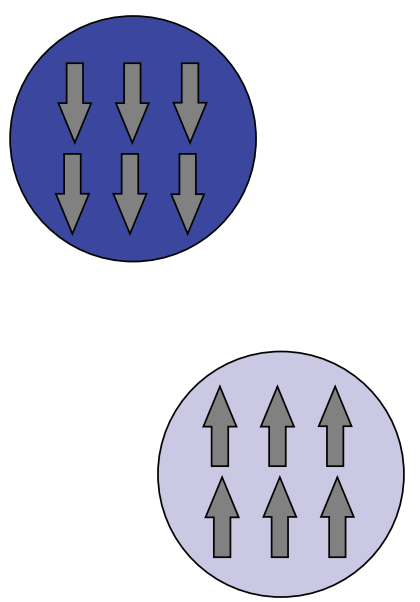

(a)

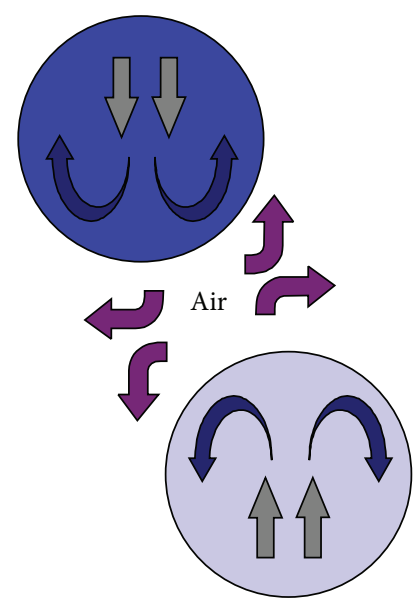

(b)

FIGURE 11: Internal flow of two drops approaching each other in (a) vacuum and (b) in air.

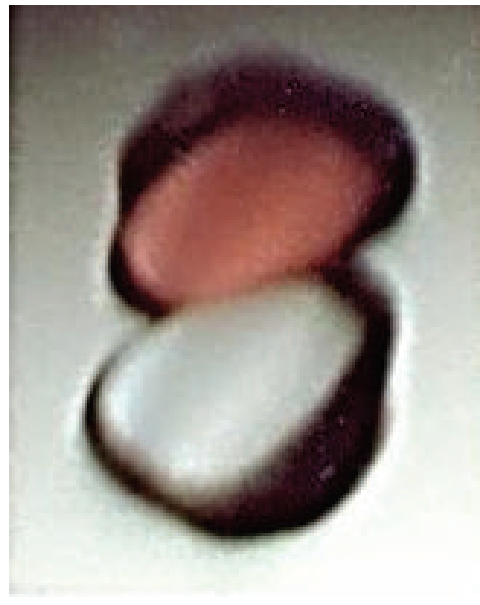

(a)

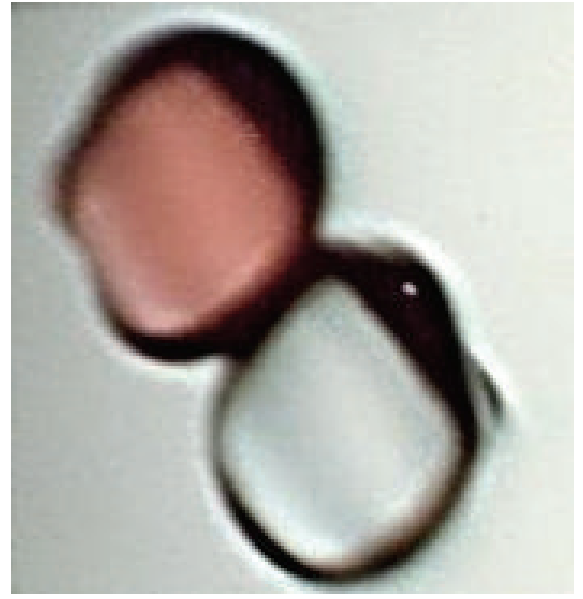

(b)

Figure 12: Two cases show the effect of trapped air in deforming the drop prior to collision and merging [unpublished photos from Ashgriz and Poo experiments [22]].

would seem to imply that, at this $(X)$ and higher impact parameters, fluid is actually pulled out of this direction. The amount of time this period takes to occur is relatively constant regardless of impact parameter.

Period II was defined to represent the action of fluid contraction of the disk formed during period I. When the variation of impact parameter is considered, the following observations can be made. First, the amount of contraction is relatively constant until a value of $\eta \sim 1.3$ is obtained at the end of this period for $X<0.3$. Essentially, this means that, for low impact parameters, the redirected fluid momentum in this direction dominates the flow which in turn causes the disk to contract inward. Second, as the impact parameter is increased until $X \sim 0.6$, more contraction occurs until a minimum value of $\eta \sim 0.75$ is reached. In this range of $0.3<X<0.6$, a competition between the amount of fluid momentum and the surface tension effect becomes evident, with the fluid momentum effect decreasing as the impact parameter is increased (i.e., due to the reduction in the interaction region). For impact parameters greater than 0.7 , this minimum deformation value is maintained. Therefore, at these high impact parameters, the amount of deformation does not change since the surface tension is now the controlling factor.

4.6. $y$-Direction (Minor Axis 2). The dimensionless deformation in the $y$-direction is provided in Figure 14. As with the $x$ direction variation, period I behavior still exists regardless of impact parameter variation. However, the amount of spread decreases as the impact parameter increases. Again, this is seen as a consequence of the reduction of the interaction region. Unlike in the $x$-direction, spread is observed up until $X \sim 0.8$ (instead of $X \sim 0.6$ as seen in the $x$-direction). At higher impact parameters, the amount of deformation shifts to contraction instead. This indicates that more mixing occurs in the $y$-direction than in the $x$-direction, since 


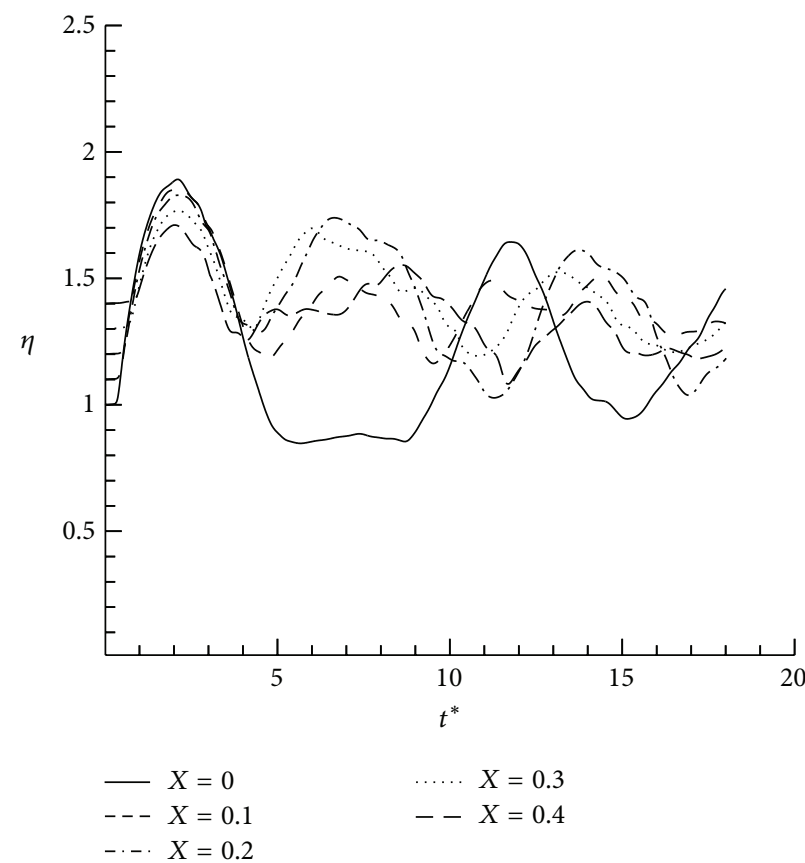

(a)

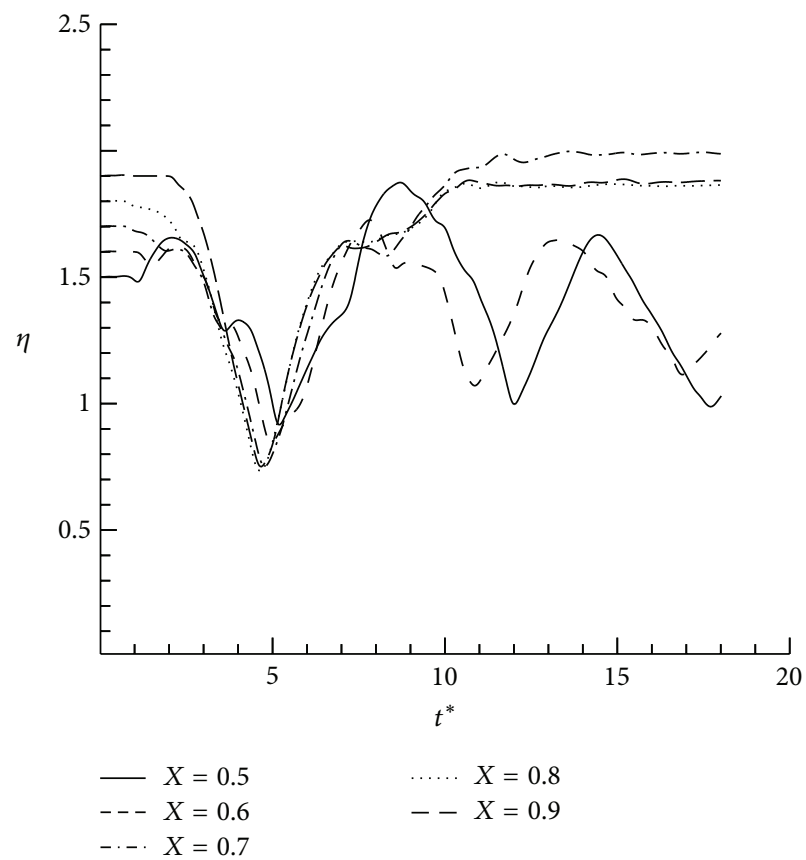

(b)

FIGURE 13: $x$-direction deformation for $\mathrm{We}=30$ and $\mathrm{Re}=810$. (a) Low impact parameters, $X<0.5$, and (b) high impact parameters, $X \geq 0.5$.

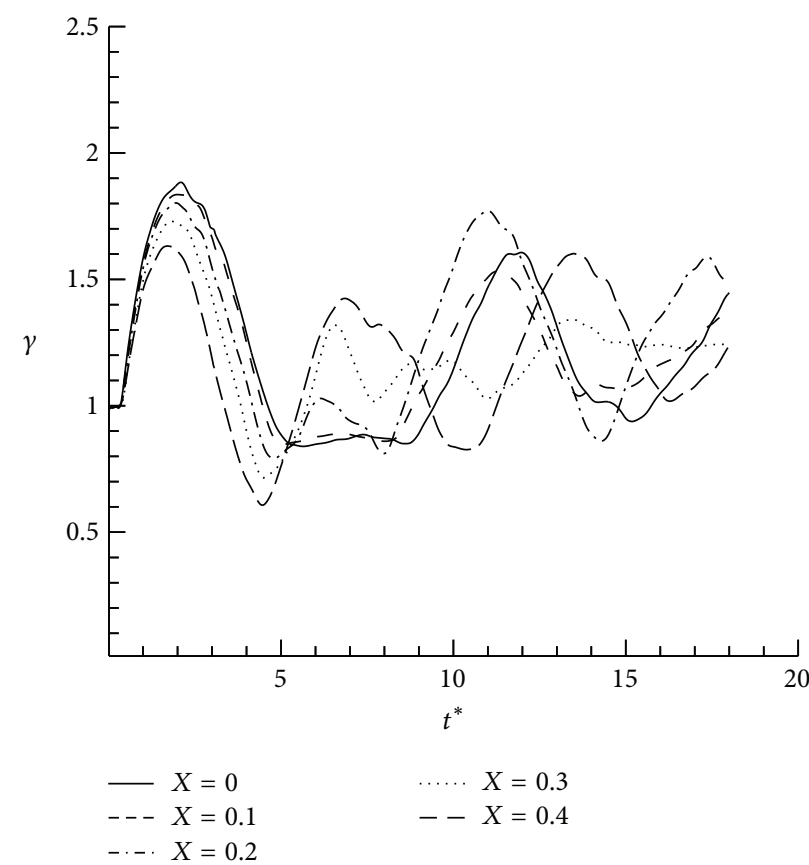

(a)

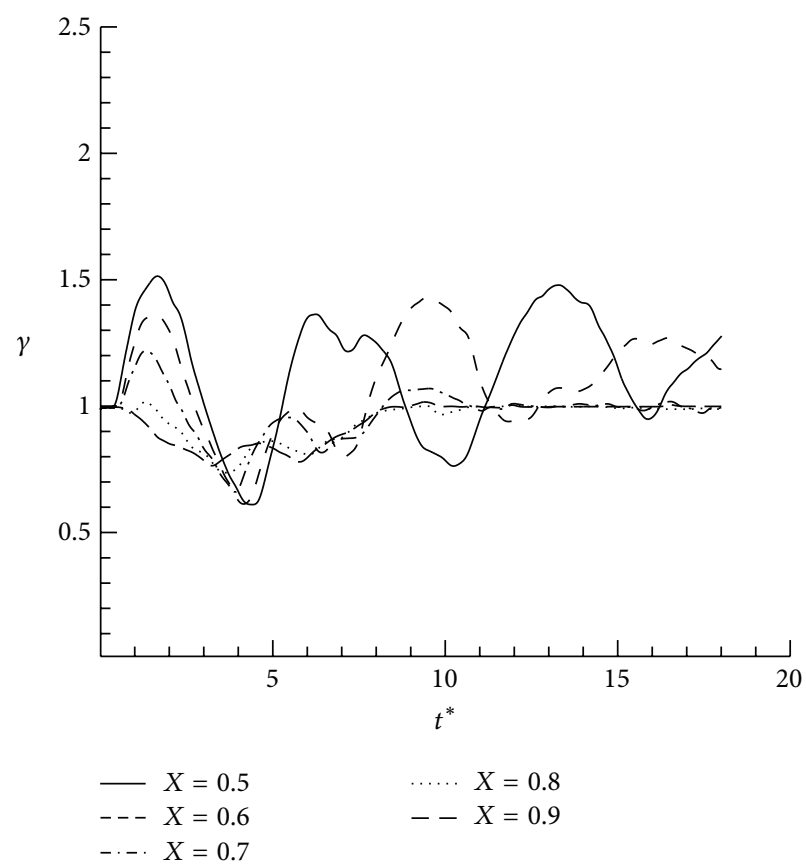

(b)

FIGURE 14: $y$-direction deformation for $\mathrm{We}=30$ and $\mathrm{Re}=810$. (a) Low impact parameters, $X<0.5$, and (b) high impact parameters, $X \geq 0.5$.

the fluid is still able to spread outward for higher impact parameters.

During period II the overall amount of contraction remains constant (since similar slopes are observed) for low impact parameters of $X<0.3$ (i.e., $\gamma$ at the end of this period decreases from 0.85 to 0.7$)$. Therefore, even though more deformation is observed at the end of this period, the control parameter is still fluid momentum as it is for the $x$-direction case. The smaller $\gamma$ values are the consequence of smaller $\gamma_{\max }$ values observed during period I. For impact parameters in 


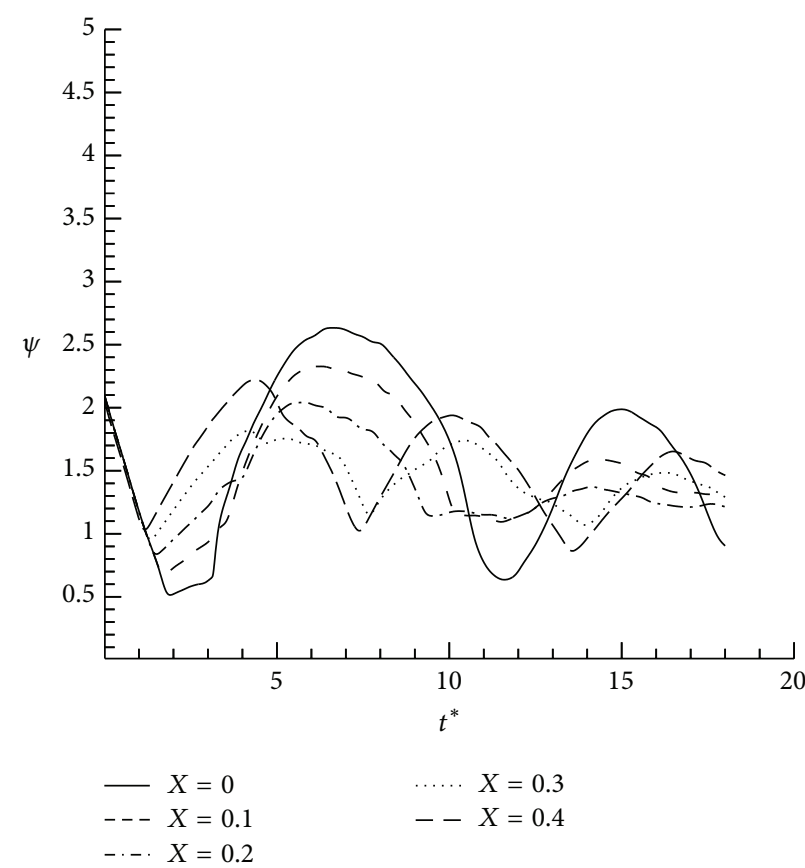

(a)

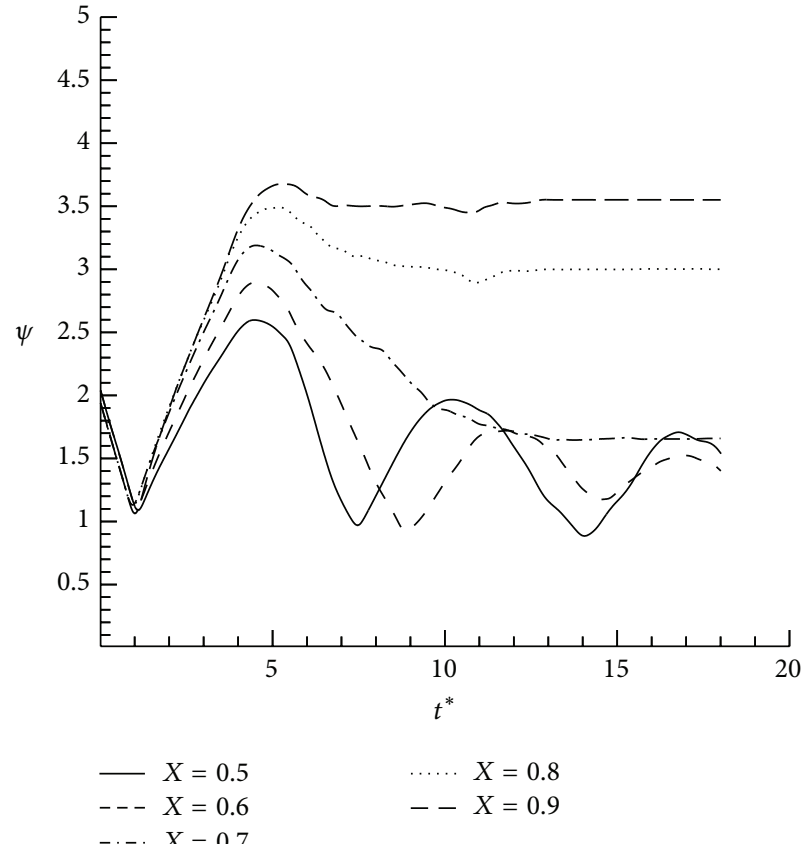

(b)

FIGURE 15: $z$-direction deformation for We $=30$ and $\mathrm{Re}=810$. (a) Low impact parameters, $X<0.5$, and (b) high impact parameters, $X \geq 0.5$.

the range of $0.4-0.6$, the amount of deformation at the end of this period is constant and has a value of $\gamma \sim 0.6$ indicating a shift in dominance from fluid momentum to surface tension. As the impact parameter is further increased, $X>0.7$, less overall deformation occurs since the amount of initial spread seen during period I has decreased and surface tension effect is in control.

4.7. $z$-Direction (Major Axis). In general, as the impact parameter increases, the region of interaction between the two drops decreases causing the intensity of deformation to shift from the $x$ - and $y$-directions to the $z$-direction. This is clearly observed in the $z$-deformation curves of Figure 15. For an impact parameter of 0 , the maximum deformation obtained is 2.7 times the drop's initial diameter and occurs at $t_{c}^{*}=6.7$. As the impact parameter is increased the maximum height obtained decreases and occurs earlier in time until an impact parameter of 0.3 is reached. At this impact parameter the maximum deformation is 1.8 times the diameter and occurs at $t_{c}^{*}=4.1$. As the impact parameter is increased further the maximum deformation increases linearly until $\psi_{\max }=3$ times the diameter is obtained. As with head-on collisions, this value defines a limiting value in which either permanent coalescence or separation occurs. This is observed at impact parameters of 0.8 and 0.9 , in which the combined mass separates by the pinching mechanism explained earlier. In the case of the impact parameter of 0.7 , the contraction rate is greater than the pinching mechanism and stretchingcoalescence is observed.

The behavior of this maximum deformation parameter, $\psi_{\max }$, as a function of impact parameter and Weber number
TABLE 2: Slopes of lines in Figure 16.

\begin{tabular}{lccc}
\hline$X$ & $m_{D=300 \mu \mathrm{m}}$ & $m_{D=700 \mu \mathrm{m}}$ & $\delta m$ \\
\hline 0.4 & 0.018 & $\mathrm{n} / \mathrm{a}$ & - \\
0.5 & 0.038 & 0.046 & 0.008 \\
0.6 & 0.043 & 0.051 & 0.008 \\
0.7 & 0.058 & 0.063 & 0.005 \\
0.8 & 0.073 & 0.078 & 0.005 \\
0.9 & 0.088 & 0.087 & 0.001 \\
0.95 & $\mathrm{n} / \mathrm{a}$ & 0.096 & - \\
\hline
\end{tabular}

is plotted in Figure 16. Figure 16(a) represents the results for collisions with drop diameters of $300 \mu \mathrm{m}$ and Figure 16(b) is for collision with diameters of $700 \mu \mathrm{m}$. Open symbols indicate the collision outcome is coalescence while solid symbols indicate separation collision. Review of this figure indicates several aspects. First, the behavior of $\psi_{\max }$ is linear over the range of Weber numbers simulated and for illustrative purposes a straight line is fitted through each set of impact data. The slope of each impact parameter data set, $m$, is computed and presented in Table 2. The subscripts 300 and 700 refer to the drop size. Also included in the table is the change in slope, $\delta m$, which indicates the difference in the slope as the drop size is decreased from $700 \mu \mathrm{m}$ to $300 \mu \mathrm{m}$.

Regardless of drop size, the slope of the lines increases with the increase in the impact parameter. This is attributed to the reduction in the interaction region which in turn causes the bulk of the fluid motion to continue traveling in the original $z$-direction. It is also interesting to note that the drop size has a small impact on the final stretch length. This is 


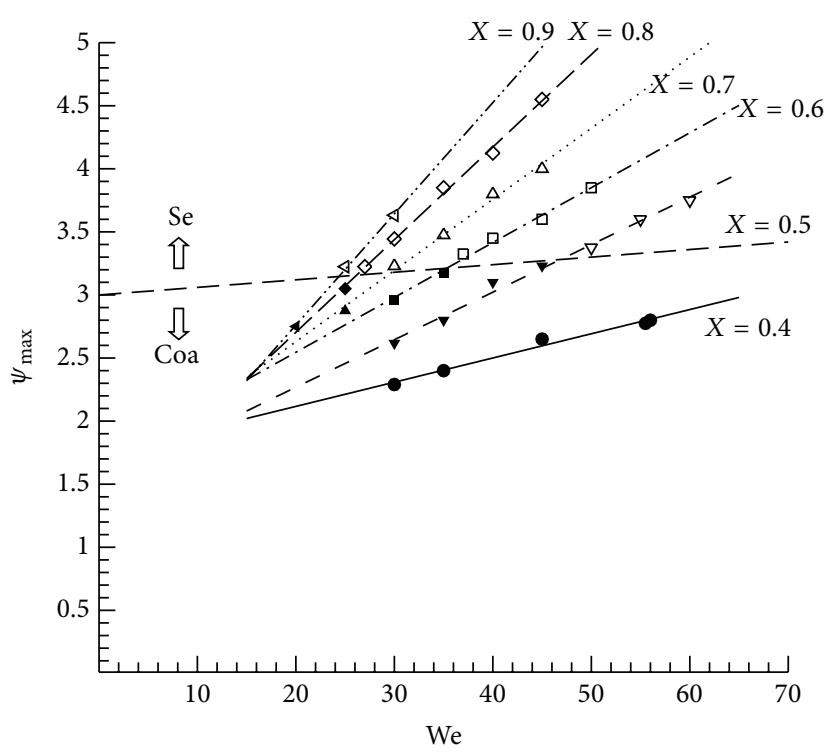

(a)

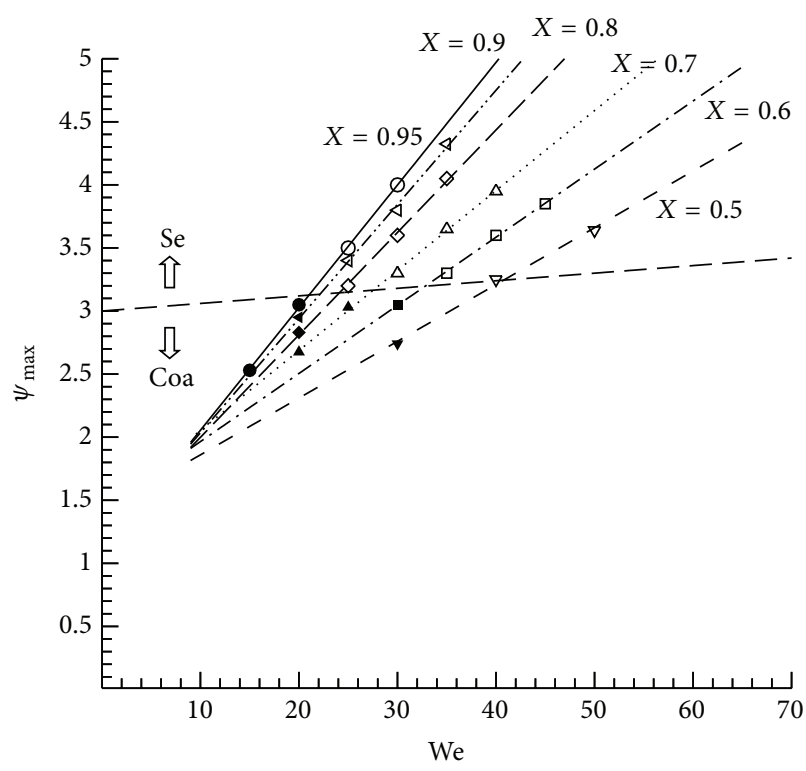

(b)

FIGURE 16: Maximum $z$-deformation for various Weber numbers and impact parameters. (a) $300 \mu \mathrm{m}$ drops and (b) $700 \mu \mathrm{m}$ drops. Solid symbols, coalescence, and open symbols, separation. Boundary line, $\psi_{\max }=0.006 \mathrm{We}+3$.

evident by the increased slope values for the $700 \mu \mathrm{m}$ drop collisions as opposed to $300 \mu \mathrm{m}$ drop collision occurring at the same impact parameter. Additionally, the slopes for $X=$ 0.9 are very similar and as a result this indicates that the size effect is no longer a factor in the overall stretching process.

A last comment concerning Figure 16 concerns the boundary line (represented by a thick dashed line) indicating the regions of coalescence and separation. According to Lord Rayleigh's capillary instability model [44], this boundary has a value of $\psi_{\max }=\pi$. However, for off-axis collisions, this boundary was empirically determined to be

$$
\psi_{\max }=0.006 \mathrm{We}+3,
$$

where the slope reflects the impact parameter effect.

4.8. Dissipation Based Models. At the beginning of the collision, both drops have a known amount of surface, $\mathrm{SE}_{o}$, and kinetic energies, $\mathrm{KE}_{o}$. These energies are computed as follows:

$$
\begin{aligned}
\mathrm{SE}_{o} & =2 \pi D_{i}^{2} \sigma, \\
\mathrm{KE}_{o} & =\frac{\pi}{24} \rho D_{i}^{3} V_{r}^{2},
\end{aligned}
$$

where the ratio of the kinetic energy to the surface energy is defined as $\mathrm{We}^{*}=\mathrm{We} / 48$ [22]. Each of these energies is computed at each time step using the following definitions:

$$
\begin{aligned}
\mathrm{SE}_{n} & =\sigma \sum_{p=1}^{N_{\text {cells }}} \mathrm{SA}_{p}, \\
\mathrm{KE}_{n} & =\frac{1}{2} \sum_{i j k=1}^{N_{\text {cells }}}\left(\frac{f_{i j k} \rho}{v_{\text {cell }}}\right)\left(u_{i j k}^{2}+v_{i j k}^{2}+w_{i j k}^{2}\right),
\end{aligned}
$$

where $\mathrm{SA}_{p}$ is the polygon area in the computational domain and $n$ is the time index, $N_{\text {cells }}$ is number of computational cells, $f_{i j k}$ is volume fraction of the liquid in the cell, and $u_{i j k}, v_{i j k}$, and $w_{i j k}$ are the velocity components in each direction. Therefore, using these relationships each energy variation is computed at each time step during the collisional process. Additionally, by using these energies and conservation of energy an estimation of the change in total energy or dissipation energy, $\phi$, is obtained as follows:

$$
\phi=\mathrm{KE}_{o}+\mathrm{SE}_{o}-\mathrm{KE}_{n}-\mathrm{SE}_{n} .
$$

The kinetic and surface energies are scaled with the initial surface energy as $\alpha=\mathrm{KE}_{n} / \mathrm{SE}_{o}$ and $\beta=\mathrm{SE}_{n} / \mathrm{SE}_{o}$. The initial surface energy is chosen as the scaling energy since for a same-size drop collision, it is independent of the impact energy. Since the amount of dissipated energy is simply the combined transient kinetic and surface energy subtracted from the initial total energy, a nondimensional dissipation energy is defined as

$$
\Phi=1-\left(\frac{\mathrm{KE}_{n}+\mathrm{SE}_{n}}{\mathrm{KE}_{o}+\mathrm{SE}_{o}}\right) .
$$

4.8.1. Kinetic Energy. The dimensionless kinetic energy variation for the binary collision of two water drops for $\mathrm{We}=$ 30 and $\operatorname{Re}=810$ is provided in Figure 17. As the impact parameter is increased, the maximum kinetic energy, $\alpha_{\max }$, decreases from 0.25 for $X=0$ to 0.22 for $0<X<$ 0.6. It should be noted that, for this range of impact parameters, the final outcome was coalescence, while higher impact parameters $(X>0.7)$ resulted in separation. This maximum kinetic energy decrease is the result of more energy conversion to surface energy (due to the increased 


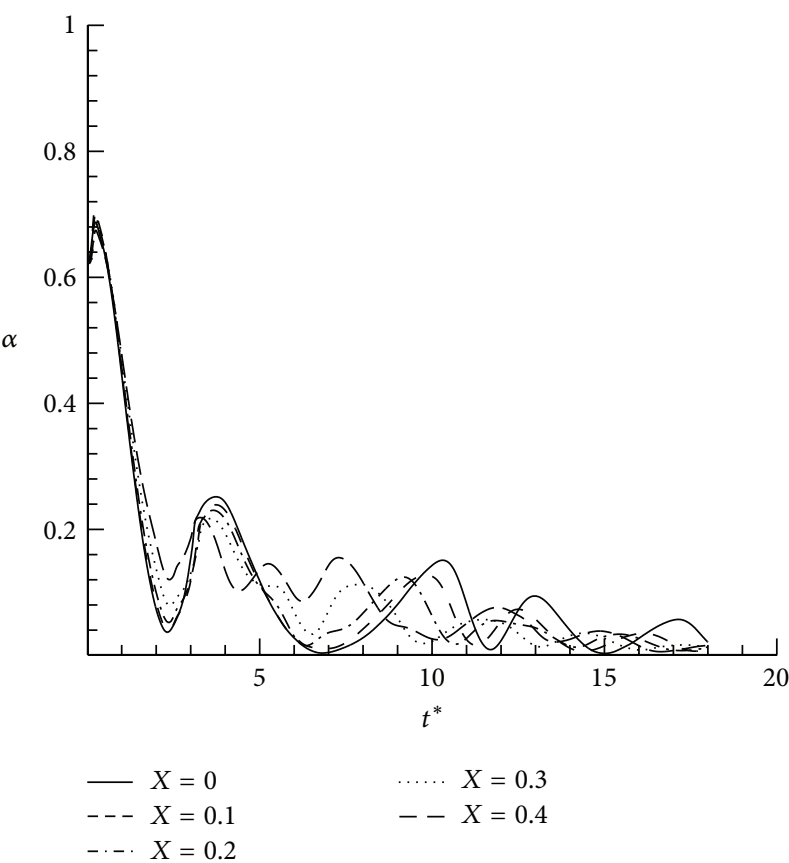

(a)

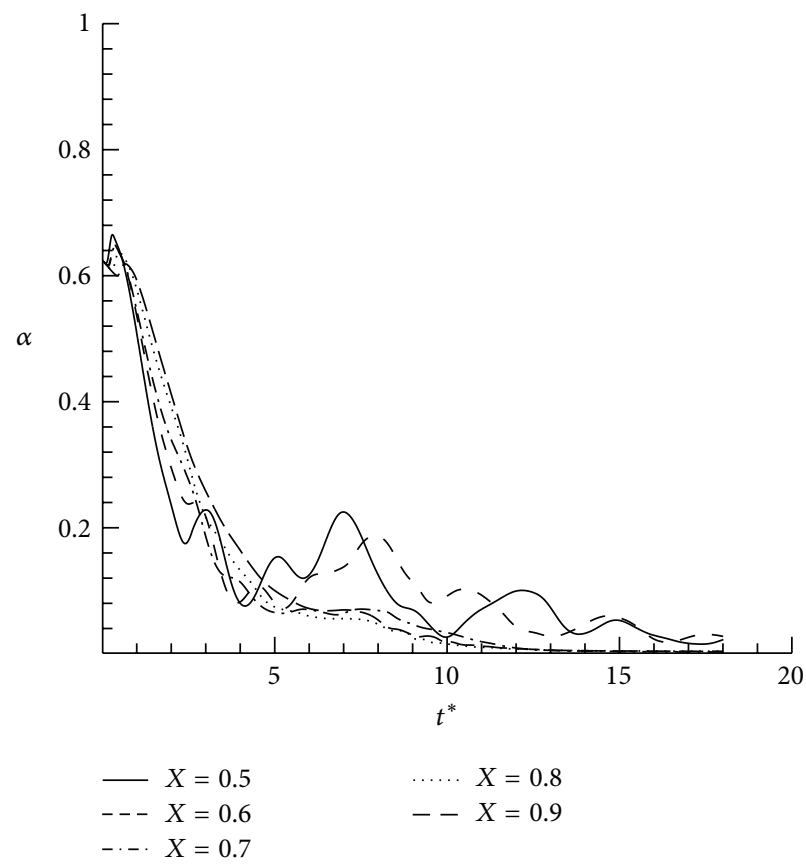

(b)

FIGURE 17: Dimensionless kinetic energy, $\alpha=\mathrm{KE}_{n} / \mathrm{SE}_{o}$ for $\mathrm{We}=30$ and $\mathrm{Re}=810$. (a) Low impact parameters, $X<0.5$, and (b) high impact parameters, $X \geq 0.5$.

surface area accompanying an increase in impact parameter). Additionally, whereas head-on collisions exhibit defined peak and valley behavior corresponding to times of maximum spread and contraction, an increase in the impact parameter causes the change in kinetic energy to undergo a more chaotic variation. It is not until the impact parameter is increased to a value greater than 0.6 that no oscillatory behavior (of any kind) is observed. Therefore, if the kinetic energy does not peak early in the evolutional sequence, separation will likely occur.

4.8.2. Surface Energy. Figure 18 provides the dimensionless surface energy variations for the binary collision case under consideration. The maximum surface energy, $\beta_{\max }$, decreases from a value of 1.325 for $X=0$ to 1.025 for the range, $0<X<0.6$. This behavior parallels the behavior seen for the kinetic energy. At impact parameters greater than 0.6 , the absence of this peak behavior at this time in the evolution is conspicuous. In fact at this time, $\beta_{\max } \sim 1$ and as noted earlier in the previous discussion, all the remaining collisions at higher impact parameters resulted in separation. Additionally, the variation in surface energy becomes more oscillatory or chaotic for impact parameters up to 0.6. At these low and intermediate impact parameters, various surface deformations occur as the combined mass tries to stabilize itself to its minimum energy state.

4.9. Reynolds Number Effect. The Reynolds number effect is presented based on the collision of two drops with impact parameters of $X=1$ and $X=0.9$. For the grazing collision $(X=1), 25$ different water drop collisions with drop sizes of $10 \mu \mathrm{m}, 75 \mu \mathrm{m}, 150 \mu \mathrm{m}, 300 \mu \mathrm{m}, 700 \mu \mathrm{m}$, and $2 \mathrm{~mm}$ are simulated and plotted in Figure 19(a). Note that the Weber and Reynolds numbers are computed using fluid properties, relative velocity, and drop size. By holding the fluid properties and relative velocity constant, the only way to produce different $\mathrm{We} / \mathrm{Re}$ combinations is to model different drop diameters. Therefore, for this range of drop diameters, the Ohnesorge number varies from 0.0370 to 0.00443 . Similarly, the results of 26 different water drop collisions with an impact parameter of 0.9 are provided in Figure 19(b).

A clear boundary between coalescence and separation is observed for both impact parameters. For the grazing collisions $(X=1)$, the boundary was produced by fitting a power function between the data points. This boundary indicates that as the Re number approached zero, the We number approached $\mathrm{We} \rightarrow 60$. This implies that all collisions with this impact parameter and a Weber number greater than 60 would result in separation. By lowering the impact parameter to 0.9 (and thus creating a region of interaction), a minimum Reynolds number between 126 and 100 is observed as the We number approaches zero. Therefore, this interacting region (which allows portions of each drop to mix) increases the viscous forces while reducing the surface tension forces, such that permanent coalescence will occur. Also, the We number approaches 22 for $X=0.9$ and 18 for $X=1$ as the Re number is increased. For head-on collision, it was shown that as the Re number increases, the We number approached $\mathrm{We} \rightarrow 35$ [40]. Therefore, the Reynolds number effect is only important for binary drop collisions with a Weber number less than 35 for $X=0$ and decreases as the impact parameter is increased. 


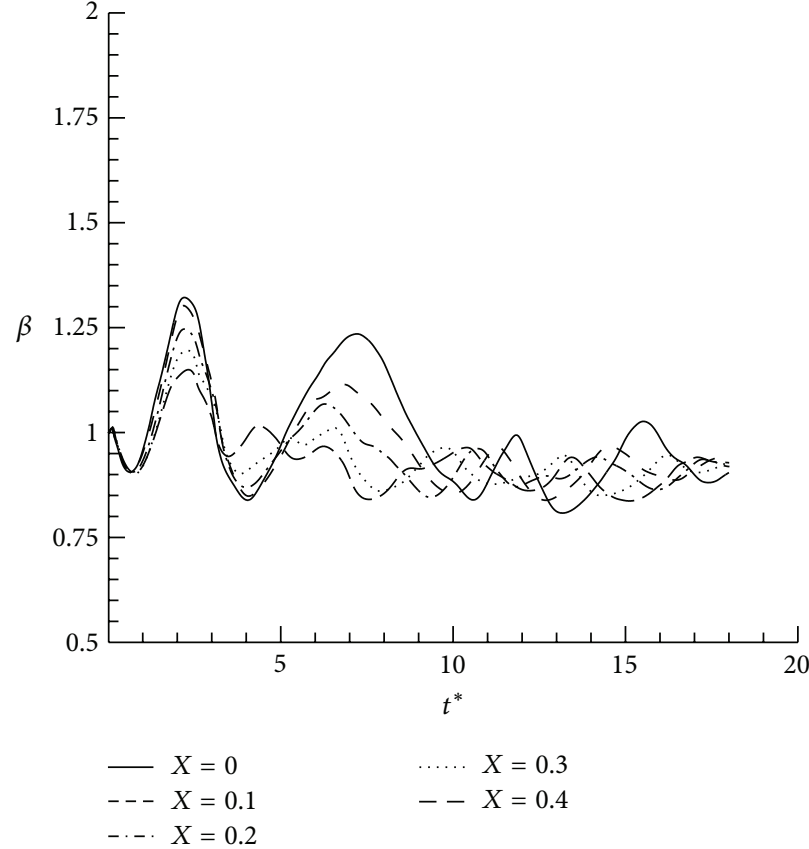

(a)

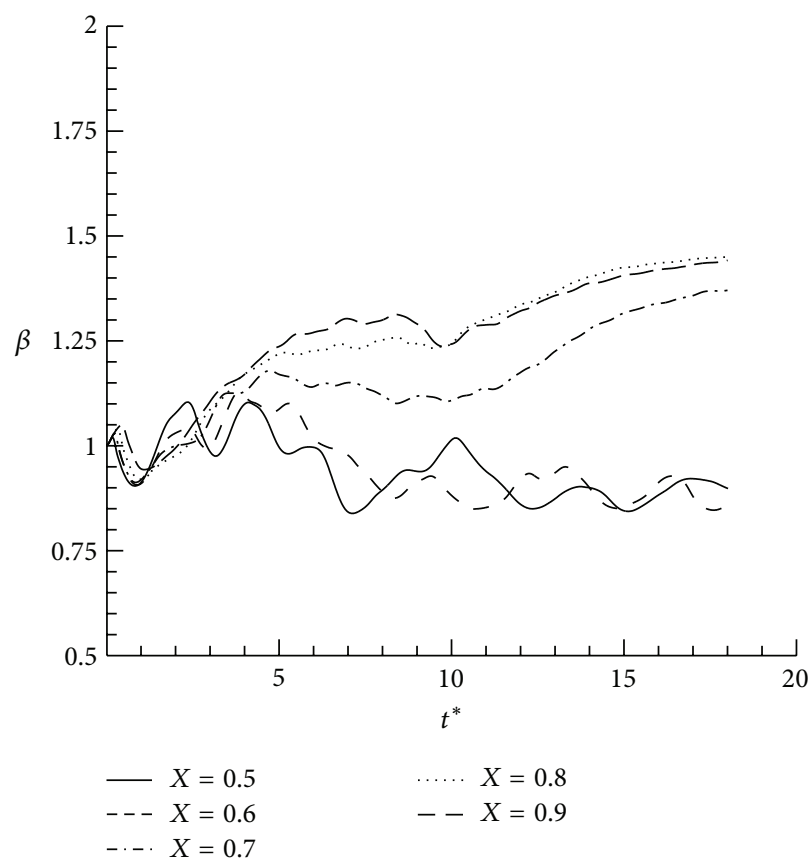

(b)

FIGURE 18: Dimensionless surface energy, $\beta=\mathrm{SE}_{n} / \mathrm{SE}_{o}$ for $\mathrm{We}=30$ and $\mathrm{Re}=810$. (a) Low impact parameters, $X<0.5$, and (b) high impact parameters, $X \geq 0.5$.

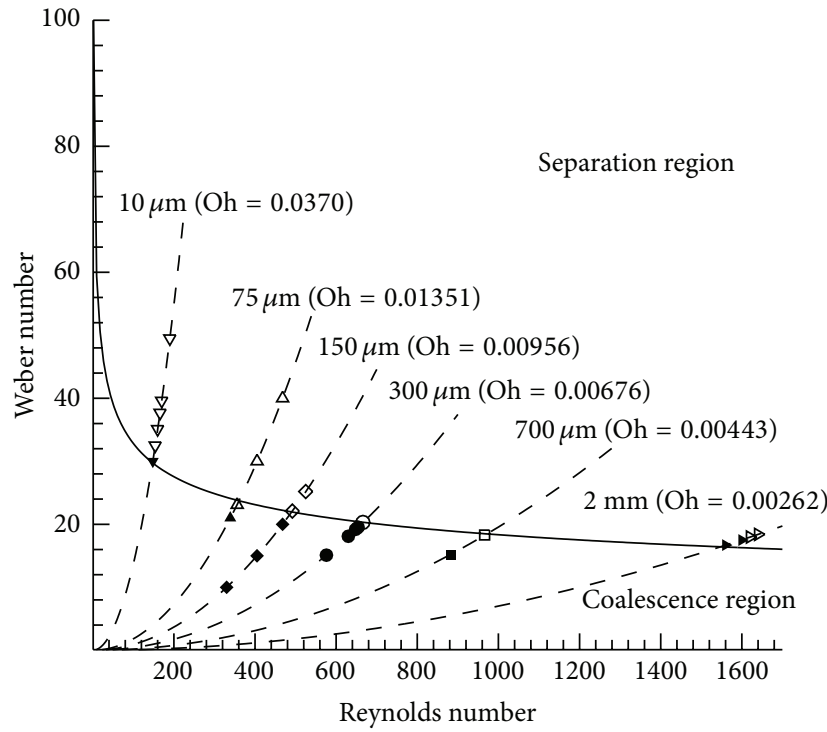

(a)

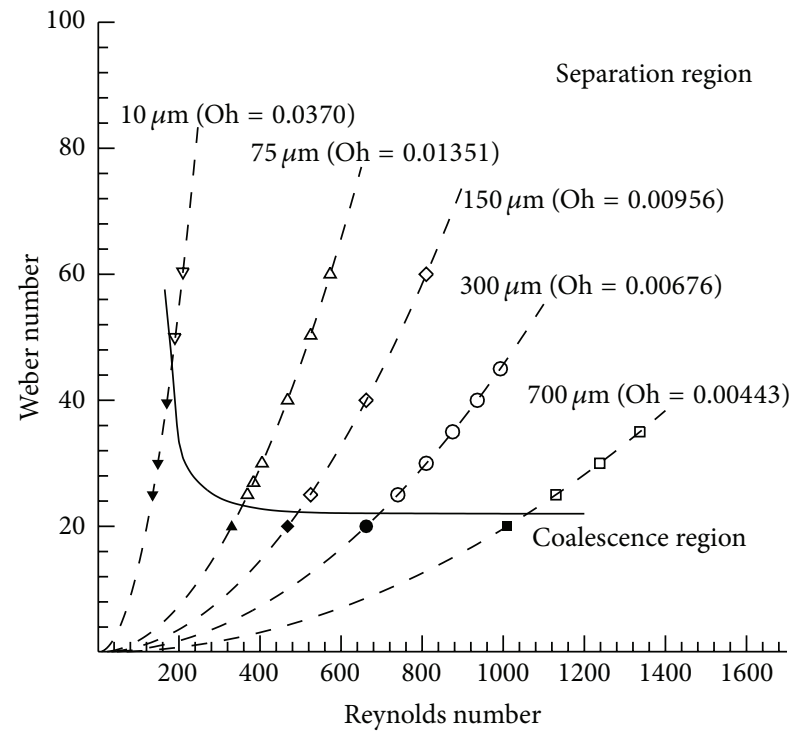

(b)

FIGURE 19: Weber versus Reynolds number. Water drop collisions. (a) $X=1$, boundary between coalescence and separation is depicted using a power fitted line. (b) $X=0.9$, in this case the boundary between coalescence and separation is shown using a spline fitted line.

\section{Conclusion}

An investigation of off-axis collisions of water drops across various impact parameters is presented. The results show that as the impact parameter is increased to values greater than zero (off-axis collision), the region of interaction between the drops is reduced. Two different types of break-up mechanisms are observed. The ligament holding the two drops may go through pinching process governed by the Rayleigh instability, or the ligament may be pulled until it is broken. The pulling (tearing) breakup occurs at high impact parameter and high Weber number combinations. In these situations, the region of interaction is insufficient to allow adequate mixing between the two colliding drops. Therefore, 
the unmixed portions of the drops continue their travel in their respective original directions, thus tearing the ligament holding the two drops.

A study of the internal flow field of droplets shows that the droplets generated after collision may have different types of internal flows. Collision with high impact parameters may result in main drops and satellite drops. The internal flow field of the main drops seems to be similar to those of the parent drops, generally unidirectional. However, the internal flow field of the satellite drops may have internal circulations and may be multidirectional. Therefore, the internal flow field of droplets in dilute sprays, which do not have significant droplet collisions, may be unidirectional. However, the internal flow fields of dense sprays, which may have significant number of high impact droplet collisions and formation of satellite droplets, may be multidirectional and have internal circulations.

The upper critical Weber number, describing the boundary between the coalescence and separation for grazing collisions $(X=1)$, is determined for water drop collisions with drop diameters ranging from $10 \mu \mathrm{m}$ to $2 \mathrm{~mm}$. These critical Weber numbers are found to be a function of the drop diameter. The smaller drops have higher We number values, that is, $\mathrm{We}_{\mathrm{cr}}=32.5$ for $D=10 \mu \mathrm{m}$, whereas $\mathrm{We}_{\mathrm{cr}}=18$ for $D=2 \mathrm{~mm}$. This is due to the higher pressures that exist in smaller drops, which quickly form a liquid bridge between the drops as soon as they touch. Smaller drops form relatively larger bridges, which requires higher impact We numbers to cause separation. However, as the drop size increases, the effect of change in drop size becomes less important. The upper critical We number approaches $\mathrm{We}=18$ as the drop size is increased. It should be noted that the above conditions are relevant for cases in which the surrounding gas is dynamically neutral, or collisions occur in vacuum. Otherwise, in determining the collision impact parameter, one has to be worried about the slippage effect that may occur due to the air entrapment in between the two drops.

A deformation criterion based on the drop deformation after collision is obtained. This criterion indicates that the drops separate if the length of the ligament formed after collision is approximately 3 times the diameter of the original drop. The maximum length of the ligament formed after the collision is found to vary linearly with the impact parameter.

Plots of the collisional outcomes in the Re-We plane are provided for the grazing collisions $(X=1)$ and collisions with an impact parameter of $X=0.9$. As the Weber number increases, the $\mathrm{Re}$ for the separation approaches zero for an impact parameter of 1 and approached $\operatorname{Re} \rightarrow 100$ for collisions with an impact parameter of 0.9. It was shown [40] that $\operatorname{Re} \rightarrow 1000$ for an impact parameter of $X=0$. Therefore, it can be concluded that collisions below this critical Reynolds number will always result in coalescence regardless of the Weber number. Also, the Reynolds number does have an effect on the collisional outcome as the impact parameter is reduced. However, this latter influence is small for drops with diameters larger than $75 \mu \mathrm{m}$ and for impact parameters of 1 and 0.9 .

\section{Conflict of Interests}

The authors declare that there is no conflict of interests regarding the publication of this paper.

\section{References}

[1] O. W. Jayaratne and B. J. Mason, "The coalescence and bouncing of water drops at an air/water interface," Proceedings of the Royal Society of London A, vol. 280, pp. 545-565, 1964.

[2] R. Gunn, "Collision characteristics of freely falling water drops," Science, vol. 150, no. 3697, pp. 695-701, 1965.

[3] D. J. Ryley and B. N. Bennett-Cowell, "The collision behaviour of steam-borne water drops," International Journal of Mechanical Sciences, vol. 9, no. 12, pp. 817-826, 1967.

[4] J. R. Adam, N. R. Lindblad, and C. D. Hendricks, "The collision, coalescence, and disruption of water droplets," Journal of Applied Physics, vol. 39, no. 11, pp. 5173-5180, 1968.

[5] R. W. Park, Behavior of water drops colliding in humid nitrogen [Ph.D. thesis], Department of Chemical Engineering, The University of Wisconsin, 1970.

[6] D. M. Whelpdale and R. List, "The Coalescence process in raindrop growth," Journal of Geophysical Research, vol. 76, no. 12, pp. 2836-2856, 1971.

[7] P. R. Brazier-Smith, S. G. Jennings, and J. Latham, "The interaction of falling water drops: coalescence," Proceedings of the Royal Society of London A, vol. 326, no. 1566, pp. 393-408, 1972.

[8] S. G. Bradley and C. D. Stow, "Collisions between liquid drops," Philosophical Transactions of the Royal Society of London A, vol. 287, no. 1349, pp. 635-678, 1978.

[9] S. G. Bradey and C. D. Stow, "On the production of satellite droplets during collisions between water drops falling in still air," Journal of the Atmospheric Sciences, vol. 36, pp. 494-500, 1979.

[10] T. B. Low and R. List, "Collision, coalescence and breakup of raindrops. Part I: experimentally established coalescence efficiencies and fragment size distributions in breakup," Journal of the Atmospheric Sciences, vol. 39, no. 7, pp. 1591-1606, 1982.

[11] T. B. Low and R. List, "Collision, coalescence and breakup of raindrops. Part II: parameterization and fragment size distributions," Journal of the Atmospheric Sciences, vol. 39, no. 7, pp. 1607-1618, 1982.

[12] V. A. Arkhipov, I. M. Vasenin, and V. F. Trofimov, "Stability of colliding drops of ideal liquid, Tomsk," Zhurnal Prikladnoi Mekhaniki i Tekhnicheskoi Fiziki, no. 3, pp. 95-98, 1983.

[13] Y. J. Jiang, A. Umemura, and C. K. Law, "An experimental investigation on the collision behaviour of hydrocarbon droplets," Journal of Fluid Mechanics, vol. 234, pp. 171-190, 1992.

[14] J. Qian and C. K. Law, "Regimes of coalescence and separation in droplet collision," Journal of Fluid Mechanics, vol. 331, pp. 5980, 1997.

[15] N. Ashgriz and P. Givi, "Binary collision dynamics of fuel droplets," International Journal of Heat and Fluid Flow, vol. 8, no. 3, pp. 205-210, 1987.

[16] N. Ashgriz and P. Givi, "Coalescence efficiencies of fuel droplets in binary collisions," International Communications in Heat and Mass Transfer, vol. 16, no. 1, pp. 11-20, 1989.

[17] G. Brenn and A. Frohn, "Collision and merging of two equal droplets of propanol," Experiments in Fluids, vol. 7, no. 7, pp. 441-446, 1989. 
[18] J.-P. Estrade, H. Carentz, G. Lavergne, and Y. Biscos, "Experimental investigation of dynamic binary collision of ethanol droplets-a model for droplet coalescence and bouncing," International Journal of Heat and Fluid Flow, vol. 20, no. 5, pp. 486-491, 1999.

[19] K. D. Willis and M. Orme, "Viscous oil droplet collisions in a vacuum," Experiments in Fluids, vol. 29, no. 4, pp. 347-358, 2000.

[20] M. Orme, "Experiments on droplet collisions, bounce, coalescence and disruption," Progress in Energy and Combustion Science, vol. 23, no. 1, pp. 65-79, 1997.

[21] A. Menchaca-Rocha, F. Huidobro, A. Martinez-Davalos et al., "Coalescence and fragmentation of colliding mercury drops," Journal of Fluid Mechanics, vol. 346, pp. 291-318, 1997.

[22] N. Ashgriz and J. Y. Poo, "Coalescence and separation in binary collisions of liquid drops," Journal of Fluid Mechanics, vol. 221, pp. 183-204, 1990.

[23] G. Brenn, S. Kalenderski, and I. Ivanov, "Investigation of the stochastic collisions of drops produced by Rayleigh breakup of two laminar liquid jets," Physics of Fluids, vol. 9, no. 2, pp. 349364, 1997.

[24] G. Brenn, D. Valkovska, and K. D. Danov, "The formation of satellite droplets by unstable binary drop collisions," Physics of Fluids, vol. 13, no. 9, pp. 2463-2477, 2001.

[25] I. V. Roisman, "Dynamics of inertia dominated binary drop collisions," Physics of Fluids, vol. 16, no. 9, pp. 3438-3449, 2004.

[26] Y. Yoon, M. Borrell, C. C. Park, and L. G. Leal, "Viscosity ratio effects on the coalescence of two equal-sized drops in a twodimensional linear flow," Journal of Fluid Mechanics, vol. 525, pp. 355-379, 2005.

[27] T.-C. Gao, R.-H. Chen, J.-Y. Pu, and T.-H. Lin, "Collision between an ethanol drop and a water drop," Experiments in Fluids, vol. 38, no. 6, pp. 731-738, 2005.

[28] S. Guido and M. Simeone, "Binary collision of drops in simple shear flow by computer-assisted video optical microscopy," Journal of Fluid Mechanics, vol. 357, pp. 1-20, 1998.

[29] M. R. Nobari, Y.-J. Jan, and G. Tryggvason, "Head-on collision of drops-a numerical investigation," Physics of Fluids, vol. 8, no. 1, pp. 29-42, 1996.

[30] S. O. Unverdi, "A front-tracking method for viscous, incompressible, multifluid flows," Journal of Computational Physics, vol. 100, no. 1, pp. 25-37, 1992.

[31] M. R. H. Nobari, Y.-J. Jan, and G. Tryggvason, "Head-on collisions of drops-a numerical investigation," Physics of Fluids, vol. 8, pp. 29-42, 1996.

[32] M. R. H. Nobari and G. Tryggvason, "Numerical simulations of three-dimensional drop collisions," AIAA Journal, vol. 34, no. 4, pp. 750-755, 1996.

[33] M. Rieber and A. Frohn, "Navier-Stokes simulation of droplet collision dynamics," in Proceedings of the 7th International Symposium on Computational Fluid Dynamic, pp. 520-525, Beijing, China, 1997.

[34] S. L. Post and J. Abraham, "Modeling the outcome of drop-drop collisions in Diesel sprays," International Journal of Multiphase Flow, vol. 28, no. 6, pp. 997-1019, 2002.

[35] F. Mashayek, N. Ashgriz, W. J. Minkowycz, and B. Shotorban, "Coalescence collision of liquid drops," International Journal of Heat and Mass Transfer, vol. 46, no. 1, pp. 77-89, 2003.

[36] Y. Morozumi, H. Ishizuka, and J. Fukai, "Criterion between permanent coalescence and separation for head-on binary droplet collision," Atomization and Sprays, vol. 15, no. 1, pp. 6180, 2005.
[37] N. Nikolopoulos, K.-S. Nikas, and G. Bergeles, "A numerical investigation of central binary collision of droplets," Computers and Fluids, vol. 38, no. 6, pp. 1191-1202, 2009.

[38] G. H. Ko and H. S. Ryou, "Modeling of droplet collisioninduced breakup process," International Journal of Multiphase Flow, vol. 31, no. 6, pp. 723-738, 2005.

[39] B. Lafaurie, C. Nardone, R. Scardovelli, S. Zaleski, and G. Zanetti, "Modelling merging and fragmentation in multiphase flows with SURFER," Journal of Computational Physics, vol. 113, no. 1, pp. 134-147, 1994.

[40] M. D. Saroka, N. Ashgriz, and M. Movassat, "Numerical investigation of head-on binary drop collisions in a dynamically inert environment," Journal of Applied Fluid Mechanics, vol. 5, no. 1, pp. 23-37, 2012.

[41] G. Brenn, "Droplet collision," in Handbook of Atomization and Sprays, N. Ashgriz, Ed., chapter 7, pp. 157-181, Springer, New York, NY, USA, 2011.

[42] C. F. Hsu and N. Ashgriz, "Impaction of a droplet on an orifice plate," Physics of Fluids, vol. 16, no. 2, pp. 400-411, 2004.

[43] N. Hsu and N. Ashgriz, "Nonlinear penetration of liquid drops into radial capillaries," Journal of Colloid and Interface Science, vol. 270, no. 1, pp. 146-162, 2004.

[44] L. Rayleigh, "On the instability of jets," Proceedings of the London Mathematical Society, vol. 10, no. 1, pp. 4-13, 1878. 

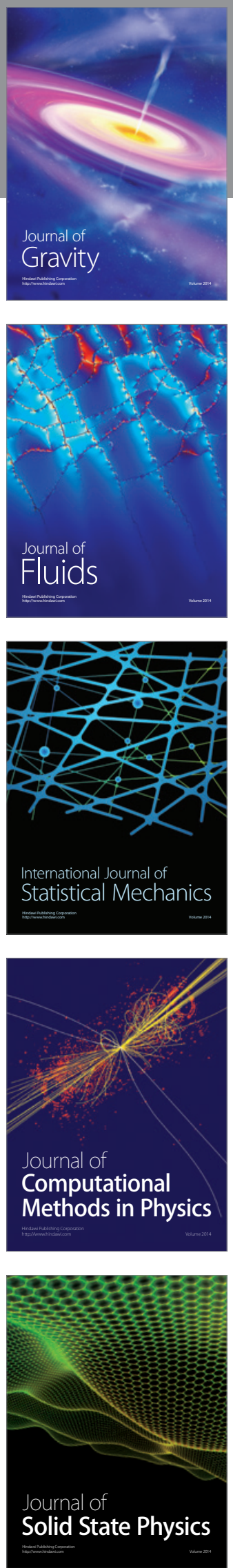

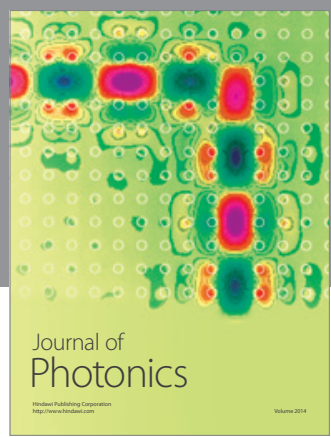

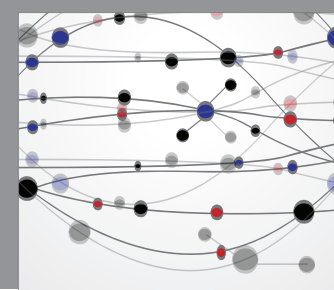

The Scientific World Journal

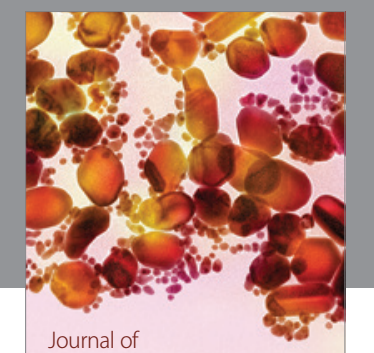

Soft Matter
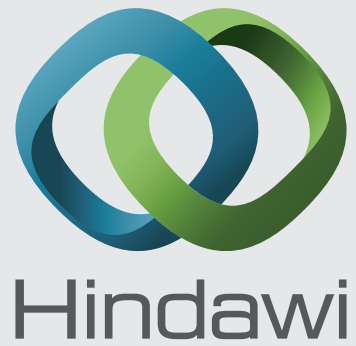

Submit your manuscripts at

http://www.hindawi.com
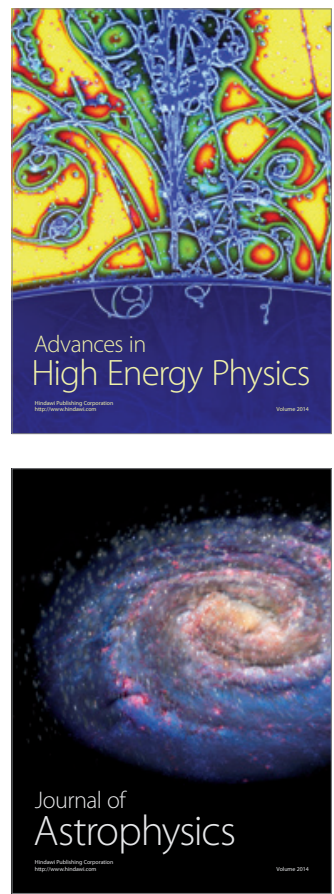
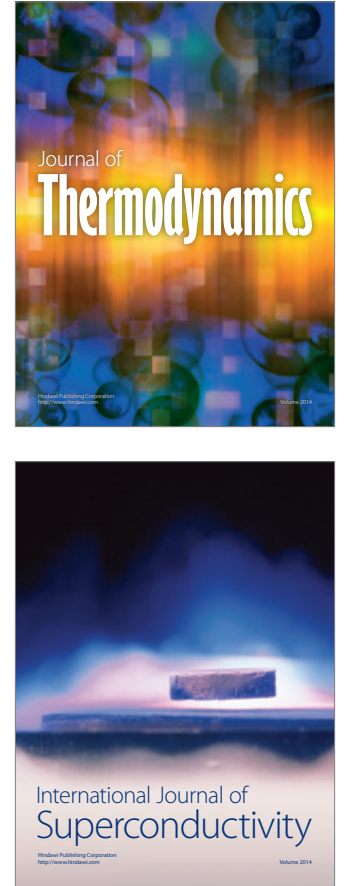
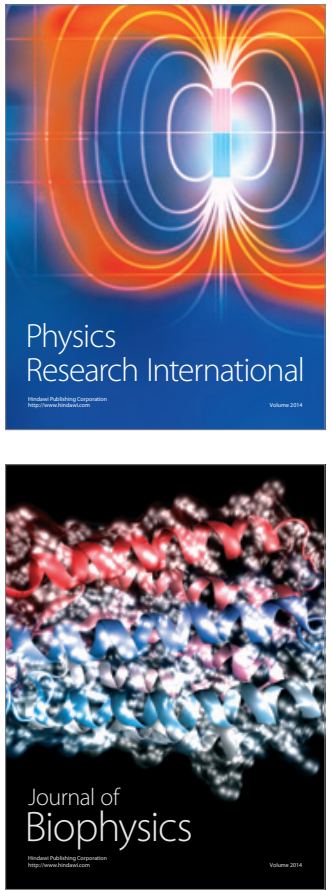
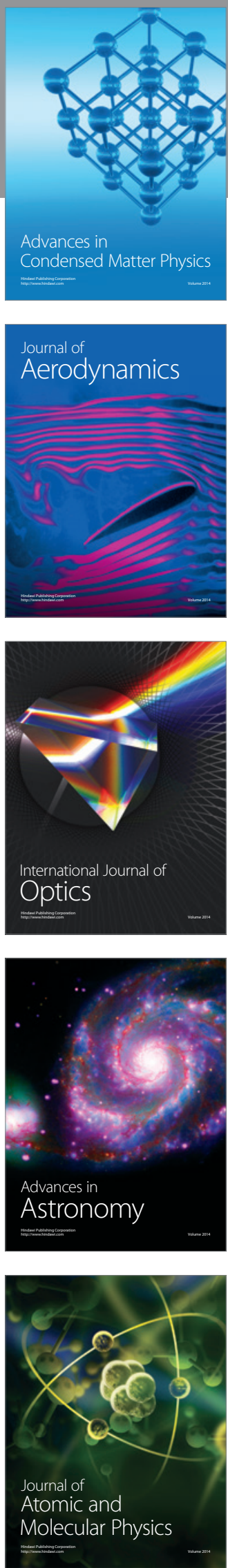